\title{
The multi-level Monte Carlo finite element method for a stochastic Brinkman Problem
}

\section{Journal Article}

\section{Author(s):}

Gittelson, Claude Jeffrey; Könnö, Juho; Schwab, Christoph; Stenberg, Rolf

Publication date:

2013-10

Permanent link:

https://doi.org/10.3929/ethz-b-000071923

\section{Rights / license:}

In Copyright - Non-Commercial Use Permitted

\section{Originally published in:}

Numerische Mathematik 125(2), https://doi.org/10.1007/s00211-013-0537-5

\section{Funding acknowledgement:}

247277 - Automated Urban Parking and Driving (EC) 


\title{
The multi-level Monte Carlo finite element method for a stochastic Brinkman Problem
}

\author{
Claude J. Gittelson • Juho Könnö • \\ Christoph Schwab • Rolf Stenberg
}

Received: 19 May 2011 / Revised: 30 November 2012 / Published online: 21 March 2013

(C) Springer-Verlag Berlin Heidelberg 2013

\begin{abstract}
We present the formulation and the numerical analysis of the Brinkman problem derived in Allaire (Arch Rational Mech Anal 113(3): 209-259,1990. doi:10. 1007/BF00375065, Arch Rational Mech Anal 113(3): 261-298, 1990. doi:10.1007/ BF00375066) with a lognormal random permeability. Specifically, the permeability is assumed to be a lognormal random field taking values in the symmetric matrices of size $d \times d$, where $d$ denotes the spatial dimension of the physical domain $D$. We prove that the solutions admit bounded moments of any finite order with respect to the random input's Gaussian measure. We present a Mixed Finite Element discretization in the physical domain $D$, which is uniformly stable with respect to the realization of the lognormal permeability field. Based on the error analysis of this mixed finite element method (MFEM), we develop a multi-level Monte Carlo (MLMC) discretization of the stochastic Brinkman problem and prove that the MLMC-MFEM allows the estimation of the statistical mean field with the same asymptotical accuracy versus work as the
\end{abstract}

C. J. Gittelson · C. Schwab $(\varangle)$

Seminar for Applied Mathematics, ETH Zurich, Rämistrasse 101, 8092 Zurich, Switzerland e-mail: schwab@math.ethz.ch

Present address:

C. J. Gittelson

Department of Mathematics, Purdue University, West Lafayette, IN 47907, USA

e-mail: cgittels@purdue.edu

\section{J. Könnö}

Wärtsilä Finland Oy, Järvikatu 2-4, 65101 Vaasa, Finland

e-mail: juho.konno@wartsila.com

R. Stenberg

Department of Mathematics and Systems Analysis, Aalto University, P.O. Box 11100, 00076Aalto, Finland

e-mail: rolf.stenberg@tkk.fi 
MFEM for a single instance of the stochastic Brinkman problem. The robustness of the MFEM implies in particular that the present analysis also covers the Darcy diffusion limit. Numerical experiments confirm the theoretical results.

Mathematics Subject Classification (2000) $\quad 65 \mathrm{C} 05 \cdot 60 \mathrm{H} 35 \cdot 65 \mathrm{~N} 30$

\section{Introduction}

Efficient numerical simulation of a viscous, incompressible flow in porous media is a key problem in the field of geosciences. It arises in an increasing number of applications related to natural resource management, environmental impact assessment and the planning and risk assessments of waste disposals. Porous media modelling is characterized by multiple scales of the permeability of the media of interest, and by uncertain geometry and material parameters of these media. We refer to [21] for a survey of porous media flow models. The present paper is devoted to a numerical analysis of a Multilevel Monte Carlo Method for a stochastic variant of the Brinkman problem. Major applications of the Brinkman model lie in petroleum engineering, in particular in the simulation of fractured reservoirs. Other industrial applications include e.g. the simulation of resin flow in composite molding and modelling of oil filters.

The Brinkman model was justified as an asymptotic limit $\varepsilon \rightarrow 0$ for media with deterministic, periodic spatial distributions of inhomogeneities of size and period $O(\varepsilon)$ in [2]. In [3], it was shown that under slightly different asymptotic scaling hypotheses, either the Stokes problem or the Darcy law is obtained in the limit $\varepsilon \rightarrow 0$. The rather delicate dependence of the limit problem on the scaling hypothesis prompted in $[15,17,18]$ the development and the numerical analysis of robust mixed FEM for a parametric class of Brinkman models which comprise, in particular, all three scaling limits obtained in [2,3]. The derivation of the Brinkman model in [2] required in particular full knowledge of the microscopic grain geometry of the porous medium in the derivation of the effective permeability tensor in the upscaled Brinkman model.

In subsurface flow models in computational geosciences, however, pore structure and geometry are not explicitly known. Accordingly, statistical hypotheses on spatial correlations of distributions of pore sizes and grain shape are made. Most widely used is the so-called log-normal distribution, in particular in connection with the (postulated valid) Darcy limit for permeability.

We therefore analyze in the present paper the Finite Element discretization of $a$ parametric, stochastic family of Brinkman models in a bounded domain $D \subset \mathbb{R}^{d}$ : a scale parameter $0 \leq t<\infty$ allows a seamless transition between the Darcy and Stokes flow, whereas the (possibly anisotropic) stochastic pore-scale geometry is assumed as a family of symmetric random $d \times d$ tensors with a log-normal law.

Robustness of the mixed FEM with respect to the scaling parameter $t$ is ensured by an error analysis in the mesh-dependent norms introduced in [15], whereas the log-normal randomness in the permeability tensors is accounted for by a Multi-Level Monte Carlo sampling strategy following [4]. 
We prove optimal convergence rates of the mixed finite element approximations of the mean velocity and pressure fields, and establish complexity bounds which show that, under realistic assumptions on the spectrum of the covariance operator for the log-normal family of permeability tensors, the multi-level Monte Carlo mixed finite element discretization allows approximating the mean velocity and pressure fields with work proportional to, essentially (i.e. up to logarithmic terms), that of a single, deterministic Brinkman solve on the finest spatial grid. In our analysis, we also exploit a discretization level dependent truncation order of the log-normal tensor's KarhunenLoève expansions, an idea that has since been taken up in [24]. We mention that the present multi-level MC approach is a so-called non-intrusive sampling strategy which is to be contrasted with recent, intrusive efforts, e.g. in [13] where a polynomial chaos type discretization for the Darcy limit was proposed and analyzed. For a general survey of theoretical properties of polynomial chaos based discretizations, we refer to [22].

Throughout the paper, we employ standard notation and terminology. The triplet $(\Omega, \mathcal{A}, \mathbb{P})$ will denote a probability space on which uncertainty is modelled. By $\mathbb{E}$ we denote the mathematical expectation with respect to the probability measure $\mathbb{P}$. The symbol $D$ will signify a bounded domain in $\mathbb{R}^{d}, d=2,3$, with a Lipschitz boundary $\partial D$. For $1 \leq p \leq \infty$, we denote by $L^{p}(D)$ the space of Lebesgue-measureable, realvalued functions in $D$ which are $p$-integrable with respect to the Lebesgue measure. For $k \in \mathbb{N}_{0}$ we denote by $H^{k}(D)$ the usual Sobolev spaces of functions in $L^{2}(D)$ whose weak derivatives of order $k$ are square integrable over $D$.

\section{The deterministic Brinkman problem}

Let $D \subset \mathbb{R}^{d}$ be a bounded Lipschitz polyhedron. The deterministic Brinkman problem on $D$ with parameters $t \geq 0$ and $\boldsymbol{M} \in L^{\infty}\left(D ; \mathbb{S}^{d}\right)$ is

$$
\left\{\begin{array}{l}
-t^{2} \boldsymbol{A u}+\boldsymbol{M u}+\nabla p=\boldsymbol{f}, \\
\operatorname{div} \boldsymbol{u}=g,
\end{array}\right.
$$

where $\boldsymbol{A} \boldsymbol{u}=\operatorname{div} \boldsymbol{\epsilon}(\boldsymbol{u})$ for $\boldsymbol{\epsilon}(\boldsymbol{u})=\left(\nabla \boldsymbol{u}+\nabla \boldsymbol{u}^{\top}\right) / 2$ and $\mathbb{S}^{d}$ denotes the space of symmetric $d \times d$ matrices, endowed with the spectral norm. We assume that $\boldsymbol{M}$ is uniformly positive definite on $D$.

For $t>0$, we assume homogeneous essential boundary conditions

$$
\boldsymbol{u}=\mathbf{0} \quad \text { on } \partial D \text {. }
$$

In the limit $t=0$, we consider the natural boundary conditions

$$
\boldsymbol{u} \cdot \boldsymbol{n}=0 \quad \text { on } \partial D
$$

Both cases require the compatibility condition $g \in L_{*}^{2}(D)$. Here and in what follows, $L_{*}^{2}(D)=L^{2}(D) / \mathbb{R}$ denotes the space of equivalence classes of functions in $L^{2}(D)$ which are equal up to a constant. We shall identify $L_{*}^{2}(D)$ in what follows with the closed subspace of $L^{2}(D)$ of functions with vanishing mean. We impose the same 
condition $p \in L_{*}^{2}(D)$ in order to ensure uniqueness of the pressure $p$ in (2.1). The solution space $\boldsymbol{V}$ for the velocity is defined as completion of $\left[C_{0}^{\infty}(D)\right]^{d}$ with respect to the norm

$$
t^{2}\|\boldsymbol{\epsilon}(\boldsymbol{v})\|_{0}^{2}+\|\boldsymbol{v}\|_{0}^{2}
$$

By Korn's inequality, this norm is equivalent (uniformly with respect to $t \geq 0$, with constants depending only on $D$ ) to

$$
\|\boldsymbol{v}\|_{t}^{2}:=t^{2}\|\nabla \boldsymbol{v}\|_{0}^{2}+\|\boldsymbol{v}\|_{0}^{2}
$$

which is the norm we use in the following. Consequently,

$$
\boldsymbol{V}=\left[H_{0}^{1}(D)\right]^{d}
$$

if $t>0$, and for $t=0$, this space is

$$
\boldsymbol{V}=\left[L^{2}(D)\right]^{d}
$$

The space for the pressure $p$ is defined through the norm

$$
\|q\|_{t}:=\sup _{\boldsymbol{v} \in \boldsymbol{V}} \frac{\langle\boldsymbol{v}, \nabla q\rangle}{\|\boldsymbol{v}\|_{t}},
$$

where $\langle\cdot, \cdot\rangle$ denotes the duality pairing in $\boldsymbol{V} \times \boldsymbol{V}^{*}$, as

$$
Q:=\left\{q \in L_{*}^{2}(D) ;\|q\|_{t}<\infty\right\} .
$$

Note that for $\boldsymbol{v} \in \boldsymbol{V}$ and $q \in Q$,

$$
\langle\boldsymbol{v}, \nabla q\rangle= \begin{cases}-(\operatorname{div} \boldsymbol{v}, q) & \text { for } t>0 \\ (\boldsymbol{v}, \nabla q) & \text { for } t=0\end{cases}
$$

where $(\cdot, \cdot)$ denotes the inner product in $L^{2}(D)^{d}$. For $t>0$, the Babuška-Brezzi property

$$
\sup _{\boldsymbol{v} \in \boldsymbol{V}} \frac{(\operatorname{div} \boldsymbol{v}, q)}{\|\boldsymbol{v}\|_{t}} \geq C\|q\|_{0} \quad \forall q \in L_{*}^{2}(D)
$$

implies that $Q=L_{*}^{2}(D)$. In the case $t=0$, we have $\|q\|_{t}=\|\nabla q\|_{0}$, and thus $Q=H_{*}^{1}(D)=H^{1}(D) \cap L_{*}^{2}(D)$.

We define the bilinear forms

$$
\begin{aligned}
& a(\boldsymbol{u}, \boldsymbol{v}):=t^{2}(\boldsymbol{\epsilon}(\boldsymbol{u}), \boldsymbol{\epsilon}(\boldsymbol{v}))+(\boldsymbol{M u}, \boldsymbol{v}) \\
& b(\boldsymbol{v}, q):=\langle\boldsymbol{v}, \nabla q\rangle
\end{aligned}
$$


and

$$
\mathcal{B}(\boldsymbol{u}, p ; \boldsymbol{v}, q):=a(\boldsymbol{u}, \boldsymbol{v})+b(\boldsymbol{v}, p)+b(\boldsymbol{u}, q) .
$$

The weak formulation of (2.1) with the boundary conditions (2.2) or (2.3) is to find $(\boldsymbol{u}, p) \in \boldsymbol{V} \times Q$ such that

$$
\mathcal{B}(\boldsymbol{u}, p ; \boldsymbol{v}, q)=\mathcal{L}(\boldsymbol{v}, q) \quad \forall(\boldsymbol{v}, q) \in \boldsymbol{V} \times Q
$$

for the linear functional

$$
\mathcal{L}(\boldsymbol{v}, q):=(\boldsymbol{f}, \boldsymbol{v})-(g, q)
$$

By Korn's inequality, uniform positive definiteness of $\boldsymbol{M}$, and (2.8), Brezzi's coercivity conditions for saddle point problems are satisfied,

$$
a(\boldsymbol{v}, \boldsymbol{v}) \geq \alpha\|\boldsymbol{v}\|_{t}^{2} \quad \forall \boldsymbol{v} \in \boldsymbol{V} \quad \text { and } \quad \sup _{\boldsymbol{v} \in \boldsymbol{V}} \frac{b(\boldsymbol{v}, q)}{\|\boldsymbol{v}\|_{t}} \geq\|q\|_{t} \quad \forall q \in Q .
$$

Furthermore, the bilinear forms $a(\cdot, \cdot)$ and $b(\cdot, \cdot)$ are bounded,

$$
\begin{aligned}
a(\boldsymbol{w}, \boldsymbol{v}) & \leq c_{a}\|\boldsymbol{w}\|_{t}\|\boldsymbol{v}\|_{t} & & \forall \boldsymbol{w}, \boldsymbol{v} \in \boldsymbol{V}, \\
b(\boldsymbol{v}, q) & \leq\|\boldsymbol{v}\|_{t}\|q\|_{t} & & \forall(\boldsymbol{v}, q) \in \boldsymbol{V} \times Q .
\end{aligned}
$$

This implies continuity of the bilinear form $\mathcal{B}(\cdot ; \cdot)$ on $[\boldsymbol{V} \times Q]^{2}$, and the stability condition

$$
\sup _{(\boldsymbol{v}, q) \in \boldsymbol{V} \times Q} \frac{\mathcal{B}(\boldsymbol{w}, r ; \boldsymbol{v}, q)}{\|\boldsymbol{v}\|_{t}+\|q\|_{t}} \geq C\left(\|\boldsymbol{w}\|_{t}+\|r\|_{t}\right) \quad \forall(\boldsymbol{w}, r) \in \boldsymbol{V} \times Q,
$$

by which the solution $(\boldsymbol{u}, p) \in \boldsymbol{V} \times Q$ exists and is unique. Moreover, we have the bounds

$$
\begin{aligned}
\|\boldsymbol{u}\|_{t} & \leq \frac{1}{\alpha}\|\boldsymbol{f}\|_{\boldsymbol{V}^{*}}+\left(1+\frac{c_{a}}{\alpha}\right)\|g\|_{Q^{*}}, \\
\|p\|_{t} & \leq\left(1+\frac{c_{a}}{\alpha}\right)\|\boldsymbol{f}\|_{V^{*}}+c_{a}\left(1+\frac{c_{a}}{\alpha}\right)\|g\|_{Q^{*}} ;
\end{aligned}
$$

see for example [6] for details. Note that the constants $\alpha$ and $c_{a}$ depend on $\boldsymbol{M}$ but not on $t$.

\section{The stochastic Brinkman problem}

We consider the matrix $\boldsymbol{M}$ in the Brinkman problem (2.1) to be a lognormal random field. To this end, let $\boldsymbol{G}$ be an $\mathbb{S}^{d}$-valued Gaussian field on $D$ with bounded paths and mean field $\boldsymbol{G}_{0}$. The distribution of $\boldsymbol{G}-\boldsymbol{G}_{0}$ is a centered Gaussian measure on 
$L^{\infty}\left(D ; \mathbb{S}^{d}\right)$, which we assume to be a Radon measure or, equivalently, to be concentrated on a separable subspace of $L^{\infty}\left(D ; \mathbb{S}^{d}\right)$, such as $C_{b}\left(D ; \mathbb{S}^{d}\right)$, see e.g. [5]. We consider $\boldsymbol{M}$ of the form

$$
\boldsymbol{M}=\exp (\boldsymbol{G})
$$

where $\exp (\cdot)$ denotes the matrix exponential. By construction, $\boldsymbol{M}$ is almost surely in $L^{\infty}\left(D ; \mathbb{S}^{d}\right)$ and uniformly positive definite.

We will denote the underlying set of elementary events by $\Omega$ and the probability measure by $\mathbb{P}$. The expectation operator $\mathbb{E}$ is the integral over $\Omega$ with respect to $\mathbb{P}$.

Lemma 3.1 For all $q>0$,

$$
\mathbb{E}\left[\exp \left(q\left\|\boldsymbol{G}-\boldsymbol{G}_{0}\right\|_{L^{\infty}(D ; \mathbb{S} d)}\right)\right]<\infty
$$

Proof By Fernique's theorem [11,5], there is a $\kappa>0$ such that

$$
\mathbb{E}\left[\exp \left(\kappa\left\|\boldsymbol{G}-\boldsymbol{G}_{0}\right\|_{L^{\infty}\left(D ; \mathbb{S}^{d}\right)}^{2}\right)\right]<\infty
$$

Consequently, for any $q>0$,

$$
\mathbb{E}\left[\exp \left(q\left\|\boldsymbol{G}-\boldsymbol{G}_{0}\right\|_{L^{\infty}\left(D ; \mathbb{S}^{d}\right)}\right)\right] \leq \exp \left(\frac{q^{2}}{4 \kappa}\right) \mathbb{E}\left[\exp \left(\kappa\left\|\boldsymbol{G}-\boldsymbol{G}_{0}\right\|_{L^{\infty}\left(D ; \mathbb{S}^{d}\right)}^{2}\right)\right]<\infty
$$

Let the random variables $\mu_{\min }$ and $\mu_{\max }$ denote the minimal and maximal eigenvalues of $\boldsymbol{M}$ on the domain $D$. By the above assumptions, $\mu_{\min }^{-1}$ and $\mu_{\max }$ are a.s. finite. Lemma 3.1 implies the following stronger property.

Proposition 3.1 For any $q \in(0, \infty), \mu_{\min }^{-1}, \mu_{\max } \in L^{q}(\Omega)$.

Proof The assertion follows from Lemma 3.1 since

$$
\mu_{\max } \leq \exp \left(\left\|\boldsymbol{G}_{0}\right\|_{L^{\infty}\left(D ; \mathbb{S}^{d}\right)}+\left\|\boldsymbol{G}-\boldsymbol{G}_{0}\right\|_{L^{\infty}\left(D ; \mathbb{S}^{d}\right)}\right)
$$

and similarly for $\mu_{\min }^{-1}$.

Together with the stability bounds (2.21) and (2.22), Proposition 3.1 leads to integrability properties of $\boldsymbol{u}$ and $p$. We note that $c_{a}$ and $\alpha$ are of the form

$$
c_{a}=\max \left(\bar{c}_{a}, \mu_{\max }\right) \text { and } \alpha=\min \left(\bar{\alpha}, \mu_{\min }\right),
$$

with $\bar{c}_{a}$ and $\bar{\alpha}$ independent of $\boldsymbol{M}$. In particular, $c_{a}$ and $\alpha^{-1}$ are also in $L^{q}(\Omega)$ for any $q \in(0, \infty)$.

Proposition 3.2 For all $q \in[1, \infty), \boldsymbol{u} \in L^{q}(\Omega ; \boldsymbol{V})$ and $p \in L^{q}(\Omega ; Q)$. 
Proof Measurability of $\boldsymbol{u}$ and $p$ follows as in [14, Lemma 3.4] by truncating the Gaussian measure such that $\boldsymbol{M}$ is uniformly bounded, formulating a sequence of wellposed saddle point problems on $L^{2}(\Omega ; \boldsymbol{V})$ and $L^{2}(\Omega ; Q)$, and passing to the limit. Suitable truncated measures can be constructed by restricting the distribution of $\boldsymbol{G}$ to a sequence of compact subsets of $L^{\infty}\left(D ; \mathbb{S}^{d}\right)$, as in [5, Theorem 3.4.1].

By (2.21) and Hölder's inequality,

$$
\|\boldsymbol{u}\|_{L^{q}(\Omega ; \boldsymbol{V})} \leq\left\|\alpha^{-1}\right\|_{L^{q}(\Omega)}\|\boldsymbol{f}\|_{\boldsymbol{V}^{*}}+\left(1+\left\|\alpha^{-1}\right\|_{L^{2 q}(\Omega)}\left\|c_{a}\right\|_{L^{2 q}(\Omega)}\right)\|g\|_{Q^{*}},
$$

and using (2.22),

$$
\begin{aligned}
\|p\|_{L^{q}(\Omega ; Q)} \leq & \left(1+\left\|\alpha^{-1}\right\|_{L^{2 q}(\Omega)}\left\|c_{a}\right\|_{L^{2 q}(\Omega)}\right)\|\boldsymbol{f}\|_{V^{*}} \\
& +\left\|c_{a}\right\|_{L^{3 q}(\Omega)}\left(1+\left\|\alpha^{-1}\right\|_{L^{3 q}(\Omega)}\left\|c_{a}\right\|_{L^{3 q}(\Omega)}\right)\|g\|_{Q^{*} .}
\end{aligned}
$$

Remark 3.1 Proposition 3.2 extends to stochastic $f$ and $g$. It follows as above that if $\boldsymbol{f} \in L^{\bar{q}}\left(\Omega ; \boldsymbol{V}^{*}\right)$ and $g \in L^{\bar{q}}\left(\Omega ; Q^{*}\right)$, then $\boldsymbol{u} \in L^{q}(\Omega ; \boldsymbol{V})$ and $p \in L^{q}(\Omega ; Q)$ for all $q \in[1, \bar{q})$. For simplicity, we consider only deterministic $f$ and $g$ in the following.

\section{Approximation by finitely many random variables}

\subsection{Truncated Gaussian field}

We approximate $\boldsymbol{M}$ by expanding the Gaussian field $\boldsymbol{G}$ in a series, and truncating this series after $N$ terms. We consider the Karhunen-Loève expansion of $\boldsymbol{G}$; however, all of the following also holds for more general series representations.

Let $\mathbb{S}_{F}^{d}$ denote $\mathbb{S}^{d}$ endowed with the Frobenius norm instead of the spectral norm. Since $L^{2}\left(D ; \mathbb{S}_{F}^{d}\right)$ is a separable Hilbert space, the covariance of $\boldsymbol{G}$ can be interpreted as a symmetric nuclear operator $K_{\boldsymbol{G}}$ on $L^{2}\left(D ; \mathbb{S}_{F}^{d}\right)$ which is given by

$$
K_{\boldsymbol{G}} \boldsymbol{A}=\mathbb{E}\left[\int_{D} \operatorname{trace}\left(\boldsymbol{A}^{\top}\left(\boldsymbol{G}-\boldsymbol{G}_{0}\right)\right) \mathrm{d} x\left(\boldsymbol{G}-\boldsymbol{G}_{0}\right)\right], \quad \boldsymbol{A} \in L^{2}\left(D ; \mathbb{S}_{F}^{d}\right) .
$$

Let $\left(\lambda_{n}\right)_{n=1}^{\infty}$ denote the eigenvalues of $K_{\boldsymbol{G}}$, and let $\left(\boldsymbol{\Phi}_{n}\right)_{n=1}^{\infty}$ be corresponding eigenvectors, normalized in $L^{2}\left(D ; \mathbb{S}_{F}^{d}\right)$. The Karhunen-Loève expansion of $\boldsymbol{G}$ is

$$
\boldsymbol{G}=\boldsymbol{G}_{0}+\sum_{n=1}^{\infty} Y_{n} \sqrt{\lambda_{n}} \boldsymbol{\Phi}_{n}
$$

where

$$
Y_{n}=\lambda_{n}^{-1 / 2} \int_{D} \operatorname{trace}\left(\left(\boldsymbol{G}-\boldsymbol{G}_{0}\right)^{\top} \boldsymbol{\Phi}_{n}\right) \mathrm{d} x
$$


are i.i.d. standard normal random variables. Let $\boldsymbol{\Psi}_{n}:=\sqrt{\lambda_{n}} \boldsymbol{\Phi}_{n}$ and

$$
\boldsymbol{G}_{N}:=\boldsymbol{G}_{0}+\sum_{n=1}^{N} Y_{n} \boldsymbol{\Psi}_{n}, \quad N \in \mathbb{N}
$$

More generally, $\left(\boldsymbol{\Psi}_{n}\right)_{n=1}^{\infty}$ may be any orthonormal basis of the Cameron-Martin space of $\boldsymbol{G}$. Since the distribution of $\boldsymbol{G}-\boldsymbol{G}_{0}$ is a centered Radon Gaussian measure on $L^{\infty}\left(D ; \mathbb{S}^{d}\right), \boldsymbol{G}_{N}$ converges to $\boldsymbol{G} \mathbb{P}$-a.s. in $L^{\infty}\left(D ; \mathbb{S}^{d}\right)$ by [5, Theorem 3.5.1]. Furthermore, convergence in $L^{q}\left(\Omega ; L^{\infty}\left(D ; \mathbb{S}^{d}\right)\right)$ for all $q \in[1, \infty)$ follows from $[5$, Corollary 3.5.8]. We make additional assumptions in order to derive a convergence rate.

We abbreviate $\psi_{n}:=\left\|\boldsymbol{\Psi}_{n}\right\|_{L^{\infty}\left(D ; \mathbb{S}^{d}\right)}$, and assume without loss of generality that $\psi:=\left(\psi_{n}\right)_{n=1}^{\infty}$ is nonincreasing. Furthermore, let $\delta_{n}:=\psi_{n}^{\kappa}\left\|D \boldsymbol{\Psi}_{n}\right\|_{L^{\infty}\left(D ; \mathcal{L}\left(\mathbb{R}^{d}, \mathbb{S}^{d}\right)\right)}$ for a $\kappa \geq 0$, and $\delta:=\left(\delta_{n}\right)_{n=1}^{\infty}$.

Assumption 4.1 The functions $\boldsymbol{\Psi}_{n}$ are in $W^{1, \infty}\left(D ; \mathbb{S}^{d}\right)$ for all $n \in \mathbb{N}$. The sequence of norms $\boldsymbol{\psi}$ is in $\ell^{\tau}$ for a $\tau \in(0,2)$, and $\delta$ is in $\ell^{\varrho}$ for a $\varrho \in[\tau, \infty]$.

Remark 4.1 Assumption 4.1 implies in particular that $\boldsymbol{G}-\boldsymbol{G}_{0} \in C_{b}\left(D ; \mathbb{S}^{d}\right)$ almost surely. Since $\boldsymbol{\Psi}_{n} \in C_{b}\left(D ; \mathbb{S}^{d}\right)$ for all $n \in \mathbb{N}, \boldsymbol{G}_{N}-\boldsymbol{G}_{0}$ is continuous for all $N$, and continuity of $\boldsymbol{G}-\boldsymbol{G}_{0}$ follows since $\boldsymbol{G}_{N}$ converges to $\boldsymbol{G}$ a.s. in $L^{\infty}\left(D ; \mathbb{S}^{d}\right)$.

Lemma 4.1 For any $q \geq \tau$ and any $N \in \mathbb{N}$,

$$
\left(\sum_{n=N+1}^{\infty} \psi_{n}^{q}\right)^{1 / q} \leq\|\psi\|_{\ell^{\tau}}(N+1)^{-s}, \quad s=\frac{1}{\tau}-\frac{1}{q} .
$$

Proof Due to the elementary estimate

$$
\|\boldsymbol{\psi}\|_{\ell^{\tau}}^{\tau}=\sum_{i=1}^{\infty} \psi_{i}^{\tau} \geq \sum_{i=1}^{n} \psi_{i}^{\tau} \geq \sum_{i=1}^{n} \psi_{n}^{\tau}=n \psi_{n}^{\tau}
$$

we have $\psi_{n} \leq n^{-1 / \tau}\|\boldsymbol{\psi}\|_{\ell^{\tau}}$ for all $n \in \mathbb{N}$. Therefore, using $q-\tau>0$,

$$
\sum_{n=N+1}^{\infty} \psi_{n}^{q} \leq \sum_{n=N+1}^{\infty} \psi_{n}^{\tau} \psi_{N+1}^{q-\tau} \leq\|\boldsymbol{\psi}\|_{\ell^{\tau}}^{\tau}(N+1)^{-(q-\tau) / \tau}\|\boldsymbol{\psi}\|_{\ell^{\tau}}^{q-\tau}=\|\boldsymbol{\psi}\|_{\ell^{\tau}}^{q}(N+1)^{-s q}
$$

for all $N \in \mathbb{N}$, with $s$ as in (4.5).

Theorem 4.1 For any $q \in[1, \infty)$ and any sufficiently small $\vartheta>0$, there is a constant $c_{q, \vartheta}$ such that for all $N \in \mathbb{N}$,

$$
\left\|\boldsymbol{G}-\boldsymbol{G}_{N}\right\|_{L^{q}\left(\Omega ; L^{\infty}\left(D ; \mathbb{S}^{d}\right)\right)} \leq c_{q, \vartheta}\|\boldsymbol{\psi}\|_{\ell^{\tau}}^{1-(1+\kappa) \vartheta}\|\boldsymbol{\delta}\|_{\ell^{\varrho}}^{\vartheta}(N+1)^{-s}
$$

with $s=\frac{1-(1+\kappa) \vartheta}{\tau}+\frac{\vartheta}{\varrho}-\frac{1}{2}$ if $\varrho<\infty$ and $s=\frac{1-(1+\kappa) \vartheta}{\tau}-\frac{1}{2}$ if $\varrho=\infty$. 
Proof Let $\ell \in\left(\mathbb{S}^{d}\right)^{*}$ with $\|\ell\|_{\left(\mathbb{S}^{d}\right)^{*}}=1, N \in \mathbb{N}$ and

$$
g:=\sum_{N+1}^{\infty} Y_{n} \ell\left(\boldsymbol{\Psi}_{n}\right)
$$

Following the proof of [7, Proposition 4], we show below that for any $q \in[1, \infty)$ and any sufficiently small $\vartheta>0$, there is a constant $\tilde{c}_{q, \vartheta}$ independent of $\ell$ and $N$ such that

$$
\|g\|_{L^{q}\left(\Omega ; L^{\infty}(D)\right)} \leq \tilde{c}_{q, \vartheta}\|\boldsymbol{\psi}\|_{\ell^{\tau}}^{1-(1+\kappa) \vartheta}\|\boldsymbol{\delta}\|_{\ell \varrho}^{\vartheta}(N+1)^{-s}
$$

with $s$ as above. This shows the claim since $\mathbb{S}^{d}$ is isomorphic to $\mathbb{R}^{k}$ with $k=d(d+1) / 2$ equipped with the $\ell^{q}\left(\mathbb{R}^{k}\right)$ norm, and thus there exist $k$ continuous linear functionals $\ell_{i} \in\left(\mathbb{S}^{d}\right)^{*}$ with $\left\|\ell_{i}\right\|_{\left(\mathbb{S}^{d}\right)^{*}}=1$ such that

$$
\|\boldsymbol{A}\|_{\mathbb{S}^{d}} \leq C_{q}\left(\sum_{i=1}^{k}\left|\ell_{i}(\boldsymbol{A})\right|^{q}\right)^{1 / q} \quad \forall \boldsymbol{A} \in \mathbb{S}^{d} .
$$

Consequently,

$$
\begin{aligned}
\mathbb{E}\left[\left\|\boldsymbol{G}-\boldsymbol{G}_{N}\right\|_{L^{\infty}\left(D ; \mathbb{S}^{d}\right)}^{q}\right] & \leq C_{q}^{q} \mathbb{E}\left[\sum_{i=1}^{k}\left\|\ell_{i}\left(\boldsymbol{G}-\boldsymbol{G}_{N}\right)\right\|_{L^{\infty}(D)}^{q}\right] \\
& =C_{q}^{q} \sum_{i=1}^{k}\left\|\ell_{i}\left(\boldsymbol{G}-\boldsymbol{G}_{N}\right)\right\|_{L^{q}\left(\Omega ; L^{\infty}(D)\right)}^{q},
\end{aligned}
$$

and (4.6) follows by applying (4.8) independently to each summand.

Using that $\left(Y_{n}\right)_{n=1}^{\infty}$ are mutually uncorrelated and have unit variance, for any $x, y \in D$,

$$
\begin{aligned}
\mathbb{E}\left[(g(x)-g(y))^{2}\right] & =\mathbb{E}\left[\left(\sum_{n=N+1}^{\infty} Y_{n} \ell\left(\boldsymbol{\Psi}_{n}(x)-\boldsymbol{\Psi}_{n}(y)\right)\right)^{2}\right] \\
& =\sum_{n=N+1}^{\infty}\left(\ell\left(\boldsymbol{\Psi}_{n}(x)-\boldsymbol{\Psi}_{n}(y)\right)\right)^{2} .
\end{aligned}
$$

Due to $\|\ell\|_{\left(\mathbb{S}^{d}\right)^{*}}=1$ and

$$
\left\|\boldsymbol{\Psi}_{n}(x)-\boldsymbol{\Psi}_{n}(y)\right\|_{\mathbb{S}^{d}} \leq \min \left(2\left\|\boldsymbol{\Psi}_{n}\right\|_{L^{\infty}\left(D ; \mathbb{S}^{d}\right)},\left\|D \boldsymbol{\Psi}_{n}\right\|_{L^{\infty}\left(D ; \mathcal{L}\left(\mathbb{R}^{d}, \mathbb{S}^{d}\right)\right)}|x-y|\right),
$$

for any $\vartheta \in[0,1]$ and every $x, y \in D$, we have

$$
\left|\ell\left(\boldsymbol{\Psi}_{n}(x)-\boldsymbol{\Psi}_{n}(y)\right)\right| \leq 2^{1-\vartheta}\left\|\boldsymbol{\Psi}_{n}\right\|_{L^{\infty}\left(D ; \mathbb{S}^{d}\right)}^{1-\vartheta}\left\|D \boldsymbol{\Psi}_{n}\right\|_{L^{\infty}\left(D ; \mathcal{L}\left(\mathbb{R}^{d}, \mathbb{S}^{d}\right)\right)}^{\vartheta}|x-y|^{\vartheta} .
$$


Consequently,

$$
\begin{aligned}
& \mathbb{E}\left[(g(x)-g(y))^{2}\right] \\
& \quad \leq\left(2^{2(1-\vartheta)} \sum_{n=N+1}^{\infty}\left\|\boldsymbol{\Psi}_{n}\right\|_{L^{\infty}\left(D ; \mathbb{S}^{d}\right)}^{2(1-\vartheta)}\left\|D \boldsymbol{\Psi}_{n}\right\|_{L^{\infty}\left(D ; \mathcal{L}\left(\mathbb{R}^{d}, \mathbb{S}^{d}\right)\right)}^{2 \vartheta}\right)|x-y|^{2 \vartheta},
\end{aligned}
$$

which is independent of $\ell \in\left(\mathbb{S}^{d}\right)^{*}$. By definition of $\psi_{n}$ and $\delta_{n}$, the above sum is equal to

$$
\sum_{n=N+1}^{\infty} \psi_{n}^{2(1-(1+\kappa) \vartheta)} \delta_{n}^{2 \vartheta} \leq\left(\sum_{n=N+1}^{\infty} \psi_{n}^{2(1-(1+\kappa) \vartheta) p}\right)^{1 / p}\left(\sum_{n=N+1}^{\infty} \delta^{\varrho}\right)^{2 \vartheta / \varrho}
$$

with $p=\varrho /(\varrho-2 \vartheta)$, if $\vartheta>0$ is sufficiently small. Lemma 4.1 implies

$$
\left(\sum_{n=N+1}^{\infty} \psi_{n}^{2(1-(1+\kappa) \vartheta) p}\right)^{1 / p} \leq\|\boldsymbol{\psi}\|_{\ell^{\tau}}^{2(1-(1+\kappa) \vartheta)}(N+1)^{-2 s}
$$

with $s$ as in the statement of the theorem. This shows the estimate

$$
\mathbb{E}\left[(g(x)-g(y))^{2}\right] \leq 2^{2(1-\vartheta)}\|\boldsymbol{\psi}\|_{\ell^{\tau}}^{2(1-(1+\kappa) \vartheta)}\|\boldsymbol{\delta}\|_{\ell^{\varrho}}^{2 \vartheta}(N+1)^{-2 s}|x-y|^{2 \vartheta}
$$

for almost all $x, y \in D$.

For any $q \in[1, \infty)$ there is a constant $c_{q}$ such that for all centered Gaussian random variables $X$,

$$
\left(\mathbb{E}\left[|X|^{q}\right]\right)^{1 / q} \leq c_{q}\left(\mathbb{E}\left[|X|^{2}\right]\right)^{1 / 2}
$$

since for $\sigma=\left(\mathbb{E}\left[|X|^{2}\right]\right)^{1 / 2}$,

$\mathbb{E}\left[|X|^{q}\right]=\frac{1}{\sqrt{2 \pi} \sigma} \int_{-\infty}^{\infty}|x|^{q} \exp \left(\frac{-x^{2}}{2 \sigma^{2}}\right) \mathrm{d} x=\frac{\sigma^{q}}{\sqrt{2 \pi}} \int_{-\infty}^{\infty}|y|^{q} \exp \left(\frac{-y^{2}}{2}\right) \mathrm{d} y=c_{q}^{q} \sigma^{q}$.

Therefore, as $g(x)-g(y)$ is a centered Gaussian random variable,

$$
\mathbb{E}\left[|g(x)-g(y)|^{q}\right] \leq 2^{q(1-\vartheta)} c_{q}^{q}\|\boldsymbol{\psi}\|_{\ell^{\tau}}^{q(1-(1+\kappa) \vartheta)}\|\delta\|_{\ell \varrho}^{q \vartheta}(N+1)^{-q s}|x-y|^{q \vartheta}
$$

Let $0<v<\vartheta \leq 1$. Abbreviating $\eta:=2^{q(1-\vartheta)} c_{q}^{q}\|\boldsymbol{\psi}\|_{\ell^{\tau}}^{q(1-(1+\kappa) \vartheta)}\|\delta\|_{\ell \varrho}^{q \vartheta}(N+1)^{-q s}$, we have

$$
\mathbb{E}\left[|g|_{W^{v, q}(D)}^{q}\right]=\mathbb{E}\left[\int_{D} \int_{D} \frac{|g(x)-g(y)|^{q}}{|x-y|^{d+q v}} \mathrm{~d} y \mathrm{~d} x\right] \leq \eta \int_{D} \int_{D}|x-y|^{q(\vartheta-v)-d} \mathrm{~d} y \mathrm{~d} x,
$$


and the latter integral is finite since $D$ is bounded. Similarly, recalling the definition (4.7) of $g$, we have for any $x \in D$,

$$
\mathbb{E}\left[g(x)^{2}\right]=\sum_{n=N+1}^{\infty} \ell\left(\boldsymbol{\Psi}_{\boldsymbol{n}}\right)^{2} \leq \sum_{n=N+1}^{\infty} \psi_{n}^{2} \leq\|\boldsymbol{\psi}\|_{\ell^{\tau}}^{2}(N+1)^{-2 \bar{s}},
$$

where $\bar{s}:=\tau^{-1}-1 / 2>s$. Since $g(x)$ is a centered Gaussian random variable,

$$
\left(\mathbb{E}\left[|g(x)|^{q}\right]\right)^{1 / q} \leq c_{q}\left(\mathbb{E}\left[|g(x)|^{2}\right]\right)^{1 / 2} \leq c_{q}\|\boldsymbol{\psi}\|_{\ell^{\tau}}(N+1)^{-\bar{s}}
$$

for any $q \in[1, \infty)$. Integrating over $D$, it follows that

$$
\mathbb{E}\left[\|g\|_{L^{q}(D)}^{q}\right] \leq|D| c_{q}^{q}\|\boldsymbol{\psi}\|_{\ell^{\tau}}^{q}(N+1)^{-q \bar{s}} \leq C \eta .
$$

Finally, we choose $q>d / v$, such that $W^{v, q}(D)$ embeds continuously into $C_{b}(D)$, see e.g. [1, Thm. 7.3.4]. Then

$$
\mathbb{E}\left[\|g\|_{L^{\infty}(D)}^{q}\right] \leq C\left(\mathbb{E}\left[\|g\|_{L^{q}(D)}^{q}\right]+\mathbb{E}\left[|g|_{W^{v, q}(D)}^{q}\right]\right) \leq C \eta,
$$

which shows (4.8) for $q>d / v$. For smaller $q$, (4.8) follows by Jensen's inequality.

Remark 4.2 We note that the convergence rate in Theorem 4.1 is independent of $q$, and essentially independent of the summability $\varrho$ of $\delta$ and the parameter $\kappa$ in the definition of $\delta$. For any $q \in[1, \infty)$ and any $0<s<\bar{s}:=\tau^{-1}-1 / 2$,

$$
\left\|\boldsymbol{G}-\boldsymbol{G}_{N}\right\|_{L^{q}\left(\Omega ; L^{\infty}\left(D ; \mathbb{S}^{d}\right)\right)} \leq C N^{-s}, \quad N \in \mathbb{N} .
$$

4.2 Truncation error in the log-Gaussian field

We define a sequence of approximations to the log-Gaussian field $\boldsymbol{M}$ by

$$
\boldsymbol{M}_{N}:=\exp \left(\boldsymbol{G}_{N}\right)=\exp \left(\boldsymbol{G}_{0}+\sum_{n=1}^{N} Y_{n} \boldsymbol{\Psi}_{n}\right), \quad N \in \mathbb{N}
$$

By continuity of the matrix exponential, $\boldsymbol{M}_{N}$ converges to $\boldsymbol{M}$ a.s. in $L^{\infty}\left(D ; \mathbb{S}^{d}\right)$. We show that convergence also holds in $L^{q}\left(\Omega ; L^{\infty}\left(D ; \mathbb{S}^{d}\right)\right)$, with the same rate as in the convergence of $\boldsymbol{G}_{N}$ to $\boldsymbol{G}$.

Lemma 4.2 For all $q \in[1, \infty)$ there is a constant $B_{q}$ such that

$$
\begin{gathered}
\left\|\exp \left(\left\|\boldsymbol{G}_{N}-\boldsymbol{G}_{0}\right\|_{L^{\infty}\left(D ; \mathbb{S}^{d}\right)}\right)\right\|_{L^{q}(\Omega)} \leq B_{q} \quad \forall N \in \mathbb{N}, \\
\left\|\exp \left(\left\|\boldsymbol{G}-\boldsymbol{G}_{0}\right\|_{L^{\infty}\left(D ; \mathbb{S}^{d}\right)}\right)\right\|_{L^{q}(\Omega)} \leq B_{q} .
\end{gathered}
$$


Proof The claim is a consequence of Fernique's theorem, see [5, Theorem 2.8.5] and [7, Proposition 7]. Since $\boldsymbol{G}_{N}-\boldsymbol{G}_{0}$ converges to $\boldsymbol{G}-\boldsymbol{G}_{0}$ in $L^{2}\left(\Omega ; L^{\infty}\left(D ; \mathbb{S}^{d}\right)\right)$, there is a constant $b$ such that

$$
\left\|\boldsymbol{G}-\boldsymbol{G}_{0}\right\|_{L^{2}\left(\Omega ; L^{\infty}\left(D ; \mathbb{S}^{d}\right)\right)} \leq b, \quad\left\|\boldsymbol{G}_{N}-\boldsymbol{G}_{0}\right\|_{L^{2}\left(\Omega ; L^{\infty}\left(D ; \mathbb{S}^{d}\right)\right)} \leq b \quad \forall N \in \mathbb{N} .
$$

By Chebyshev's inequality, for any $\beta>0$ and any $N \in \mathbb{N}$,

$$
\mathbb{P}\left(\left\|\boldsymbol{G}_{N}-\boldsymbol{G}_{0}\right\|_{L^{\infty}\left(D ; \mathbb{S}^{d}\right)}>\beta\right) \leq \beta^{-2}\left\|\boldsymbol{G}_{N}-\boldsymbol{G}_{0}\right\|_{L^{2}\left(\Omega ; L^{\infty}\left(D ; \mathbb{S}^{d}\right)\right)} \leq \beta^{-2} b^{2},
$$

and the same bound holds for $\boldsymbol{G}$ in place of $\boldsymbol{G}_{N}$. For $\beta>\sqrt{2} b$, Fernique's theorem provides a constant $B_{0}$ depending only on $\beta$ and $b$ such that

$$
\mathbb{E}\left[\exp \left(\lambda\left\|\boldsymbol{G}_{N}-\boldsymbol{G}_{0}\right\|_{L^{\infty}\left(D ; \mathbb{S}^{d}\right)}^{2}\right)\right] \leq B_{0} \quad \forall N \in \mathbb{N},
$$

with $\lambda=\frac{1}{24 \beta} \log \left(\frac{\beta^{2}}{b^{2}}-1\right)$, and thus

$$
\mathbb{E}\left[\exp \left(q\left\|\boldsymbol{G}_{N}-\boldsymbol{G}_{0}\right\|_{L^{\infty}\left(D ; \mathbb{S}^{d}\right)}\right)\right] \leq \exp \left(\frac{1}{4 \lambda} q^{2}\right) B_{0}:=B_{q}^{q} \quad \forall N \in \mathbb{N}
$$

As above, the same estimate holds for $\boldsymbol{G}$ in place of $\boldsymbol{G}_{N}$.

Proposition 4.1 For any $q \in[1, \infty)$ and any sufficiently small $\vartheta>0$, there is a constant $C_{q, \vartheta}$ such that for all $N \in \mathbb{N}$,

$$
\left\|\boldsymbol{M}-\boldsymbol{M}_{N}\right\|_{L^{q}\left(\Omega ; L^{\infty}\left(D ; \mathbb{S}^{d}\right)\right)} \leq C_{q, \vartheta}\|\boldsymbol{\psi}\|_{\ell^{\tau}}^{1-(1+\kappa) \vartheta}\|\boldsymbol{\delta}\|_{\ell \varrho}^{\vartheta}(N+1)^{-s}
$$

with $s=\frac{1-(1+\kappa) \vartheta}{\tau}+\frac{\vartheta}{\varrho}-\frac{1}{2}$ if $\varrho<\infty$ and $s=\frac{1-(1+\kappa) \vartheta}{\tau}-\frac{1}{2}$ if $\varrho=\infty$.

Proof For any $\boldsymbol{A}, \boldsymbol{B} \in \mathbb{S}^{d}$, the matrix exponential satisfies

$$
\|\exp (\boldsymbol{A})-\exp (\boldsymbol{A}+\boldsymbol{B})\|_{\mathbb{S}^{d}} \leq\|\boldsymbol{B}\|_{\mathbb{S}^{d}} \exp \left(\|\boldsymbol{A}\|_{\mathbb{S}^{d}}\right) \exp \left(\|\boldsymbol{B}\|_{\mathbb{S}^{d}}\right) .
$$

Setting $\boldsymbol{A}:=\boldsymbol{G}(x)$ and $\boldsymbol{B}:=\boldsymbol{G}_{N}(x)-\boldsymbol{G}(x), x \in D$, and using the triangle inequality, we have

$$
\begin{aligned}
& \left\|\exp (\boldsymbol{G}(x))-\exp \left(\boldsymbol{G}_{N}(x)\right)\right\|_{\mathbb{S}^{d}} \\
& \quad \leq\left\|\boldsymbol{G}(x)-\boldsymbol{G}_{N}(x)\right\|_{\mathbb{S}^{d}} \exp \left(2\|\boldsymbol{G}(x)\|_{\mathbb{S}^{d}}\right) \exp \left(\left\|\boldsymbol{G}_{N}(x)\right\|_{\mathbb{S}^{d}}\right) .
\end{aligned}
$$

For $q^{-1}=\bar{q}^{-1}+3 r^{-1}$, Hölder's inequality and Lemma 4.2 imply

$$
\begin{aligned}
\left\|\boldsymbol{M}-\boldsymbol{M}_{N}\right\|_{L^{q}\left(\Omega ; L^{\infty}\left(D ; \mathbb{S}^{d}\right)\right)} & \leq\left\|\exp (\boldsymbol{G})-\exp \left(\boldsymbol{G}_{N}\right)\right\|_{L^{q}\left(\Omega ; L^{\infty}\left(D ; \mathbb{S}^{d}\right)\right)} \\
& \leq\left\|\boldsymbol{G}-\boldsymbol{G}_{N}\right\|_{L^{\bar{q}}\left(\Omega ; L^{\infty}\left(D ; \mathbb{S}^{d}\right)\right)} \exp \left(3\left\|\boldsymbol{G}_{0}\right\|_{L^{\infty}\left(D ; \mathbb{S}^{d}\right)}\right) B_{r}^{3} .
\end{aligned}
$$

Then the claim follows using Theorem 4.1 for $\bar{q}$. 
4.3 Solvability of the truncated stochastic Brinkman problem

Let $(\boldsymbol{u}, p)$ and $\left(\boldsymbol{u}_{N}, p_{N}\right)$ denote the solutions to the stochastic Brinkman problem with log-Gaussian random fields $\boldsymbol{M}$ and $\boldsymbol{M}_{N}$, respectively, and let

$$
\begin{aligned}
a(\boldsymbol{u}, \boldsymbol{v}) & :=t^{2}(\boldsymbol{\epsilon}(\boldsymbol{u}), \boldsymbol{\epsilon}(\boldsymbol{v}))+(\boldsymbol{M u}, \boldsymbol{v}), \\
a_{N}(\boldsymbol{u}, \boldsymbol{v}) & :=t^{2}(\boldsymbol{\epsilon}(\boldsymbol{u}), \boldsymbol{\epsilon}(\boldsymbol{v}))+\left(\boldsymbol{M}_{N} \boldsymbol{u}, \boldsymbol{v}\right), \quad N \in \mathbb{N},
\end{aligned}
$$

denote the bilinear forms from (2.12). Then $(\boldsymbol{u}, p)$ and $\left(\boldsymbol{u}_{N}, p_{N}\right)$ satisfy

$$
\begin{aligned}
\mathcal{B}(\boldsymbol{u}, p ; \boldsymbol{v}, q) & =\mathcal{L}(\boldsymbol{v}, q) & \forall(\boldsymbol{v}, q) & \in \boldsymbol{V} \times Q \\
\mathcal{B}_{N}\left(\boldsymbol{u}_{N}, p_{N} ; \boldsymbol{v}, q\right) & =\mathcal{L}(\boldsymbol{v}, q) & \forall(\boldsymbol{v}, q) & \in \boldsymbol{V} \times Q
\end{aligned}
$$

for the bilinear forms $\mathcal{B}(\cdot ; \cdot)$ and $\mathcal{B}_{N}(\cdot ; \cdot)$ defined by $(2.14)$ with $a(\cdot, \cdot)$ and $a_{N}(\cdot, \cdot)$ from (4.14) and (4.15), and with $\mathcal{L}(\cdot, \cdot)$ as in (2.16).

Lemma 4.3 There are random variables $\alpha, c_{a}, \alpha_{N}$ and $c_{a, N}, N \in \mathbb{N}$, such that

$$
\begin{aligned}
& a(\boldsymbol{v}, \boldsymbol{v}) \geq \alpha\|\boldsymbol{v}\|_{t}^{2}, \quad a_{N}(\boldsymbol{v}, \boldsymbol{v}) \geq \alpha_{N}\|\boldsymbol{v}\|_{t}^{2}, \quad N \in \mathbb{N}, \\
& a(\boldsymbol{w}, \boldsymbol{v}) \leq c_{a}\|\boldsymbol{w}\|_{t}\|\boldsymbol{v}\|_{t}, \quad a_{N}(\boldsymbol{w}, \boldsymbol{v}) \leq c_{a, N}\|\boldsymbol{w}\|_{t}\|\boldsymbol{v}\|_{t}, \quad N \in \mathbb{N},
\end{aligned}
$$

for all $\boldsymbol{v}, \boldsymbol{w} \in \boldsymbol{V}$. Furthermore, for any $q \in[1, \infty)$ there is a constant $A_{q}$ such that

$$
\|X\|_{L^{q}(\Omega)} \leq A_{q}
$$

uniformly for $X=\alpha^{-1}, X=c_{a}, X=\alpha_{N}^{-1}$ and $X=c_{a, N}, N \in \mathbb{N}$.

Proof By Korn's inequality, there exist constants $k$ and $K$ such that

$$
k\|\nabla \boldsymbol{v}\|_{0}^{2} \leq\|\boldsymbol{\epsilon}(\boldsymbol{v})\|_{0}^{2} \leq K\|\nabla \boldsymbol{v}\|_{0}^{2} \quad \forall \boldsymbol{v} \in \boldsymbol{V}
$$

Let the random variables $\mu_{\min }$ and $\mu_{\max }$ denote the minimal and maximal eigenvalues of $\boldsymbol{M}$ on the domain $D$, and let $\mu_{\min , N}, \mu_{\max , N}$ denote the analogous values of $\boldsymbol{M}_{N}, N \in \mathbb{N}$. Then (4.18) and (4.19) are satisfied for

$$
\begin{aligned}
& \alpha=\min \left(k, \mu_{\min }\right) \\
& c_{a}=\max \left(K, \mu_{\max }\right) \\
& \alpha_{N}=\min \left(k, \mu_{\min , N}\right) \\
& c_{a, N}=\max \left(K, \mu_{\max , N}\right) \quad \forall N \in \mathbb{N} \text {. }
\end{aligned}
$$

The estimate (4.20) follows from Lemma 4.2, using uniform boundedness of $\boldsymbol{G}_{0}$.

Using the stability estimates (2.21) and (2.22), the convergence from Theorem 4.1 and Proposition 4.1 carries over to $\left(\boldsymbol{u}_{N}, p_{N}\right)$. 
Theorem 4.2 For any $q \in[1, \infty)$ and any sufficiently small $\vartheta>0$, there are constants $C_{q, \vartheta}^{u}$ and $C_{q, \vartheta}^{p}$ such that for all $N \in \mathbb{N}$,

$$
\begin{aligned}
\left\|\boldsymbol{u}-\boldsymbol{u}_{N}\right\|_{L^{q}(\Omega ; \boldsymbol{V})} \leq C_{q, \vartheta}^{\boldsymbol{u}}\left(\|\boldsymbol{f}\|_{\boldsymbol{V}^{*}}+\|g\|_{Q^{*}}\right)\|\boldsymbol{\psi}\|_{\ell^{\tau}}^{1-(1+\kappa) \vartheta}\|\boldsymbol{\delta}\|_{\ell^{\varrho}}^{\vartheta}(N+1)^{-s} \\
\left\|p-p_{N}\right\|_{L^{q}(\Omega ; Q)} \leq C_{q, \vartheta}^{p}\left(\|\boldsymbol{f}\|_{\boldsymbol{V}^{*}}+\|g\| Q_{Q^{*}}\right)\|\boldsymbol{\psi}\|_{\ell^{\tau}}^{1-(1+\kappa) \vartheta}\|\boldsymbol{\delta}\|_{\ell^{\vartheta}}(N+1)^{-s}
\end{aligned}
$$

with $s=\frac{1-(1+\kappa) \vartheta}{\tau}+\frac{\vartheta}{\varrho}-\frac{1}{2}$ if $\varrho<\infty$ and $s=\frac{1-(1+\kappa) \vartheta}{\tau}-\frac{1}{2}$ if $\varrho=\infty$.

Proof Subtracting (4.17) from (4.16) leads to the error equation

$$
\begin{gathered}
\mathcal{B}\left(\boldsymbol{u}-\boldsymbol{u}_{N}, p-p_{N} ; \boldsymbol{v}, q\right)=\mathcal{B}_{N}\left(\boldsymbol{u}_{N}, p_{N} ; \boldsymbol{v}, q\right) \\
-\mathcal{B}\left(\boldsymbol{u}_{N}, p_{N} ; \boldsymbol{v}, q\right)=\left(\left(\boldsymbol{M}_{N}-\boldsymbol{M}\right) \boldsymbol{u}_{N}, \boldsymbol{v}\right)
\end{gathered}
$$

for all $(\boldsymbol{v}, q) \in \boldsymbol{V} \times Q$. The last term satisfies

$$
\begin{aligned}
\left(\left(\boldsymbol{M}_{N}-\boldsymbol{M}\right) \boldsymbol{u}_{N}, \boldsymbol{v}\right) & \leq\left\|\boldsymbol{M}-\boldsymbol{M}_{N}\right\|_{L^{\infty}\left(D ; \mathbb{S}^{d}\right)}\left\|\boldsymbol{u}_{N}\right\|_{\left(L^{2}(D)\right)^{d}}\|\boldsymbol{v}\|_{\left(L^{2}(D)\right)^{d}} \\
& \leq C\left\|\boldsymbol{M}-\boldsymbol{M}_{N}\right\|_{L^{\infty}\left(D ; \mathbb{S}^{d}\right)}\left\|\boldsymbol{u}_{N}\right\| \boldsymbol{V}\|\boldsymbol{v}\|_{\boldsymbol{V}} .
\end{aligned}
$$

Furthermore, (2.21) implies

$$
\left\|\boldsymbol{u}_{N}\right\|_{t} \leq \frac{1}{\alpha_{N}}\|\boldsymbol{f}\|_{V^{*}}+\left(1+\frac{c_{a, N}}{\alpha_{N}}\right)\|g\|_{Q^{*}} .
$$

Applying (2.21) and (2.22) to the error equations gives us

$$
\begin{aligned}
& \left\|\boldsymbol{u}-\boldsymbol{u}_{N}\right\|_{t} \leq C \frac{1}{\alpha}\left[\frac{1}{\alpha_{N}}\|\boldsymbol{f}\|_{V^{*}}+\left(1+\frac{c_{a, N}}{\alpha_{N}}\right)\|g\|_{Q^{*}}\right]\left\|\boldsymbol{M}-\boldsymbol{M}_{N}\right\|_{L^{\infty}\left(D ; \mathbb{S}^{d}\right)}, \\
& \left\|p-p_{N}\right\|_{t} \leq C\left(1+\frac{c_{a}}{\alpha}\right)\left[\frac{1}{\alpha_{N}}\|\boldsymbol{f}\|_{V^{*}}+\left(1+\frac{c_{a, N}}{\alpha_{N}}\right)\|g\|_{Q^{*}}\right]\left\|\boldsymbol{M}-\boldsymbol{M}_{N}\right\|_{L^{\infty}\left(D ; \mathbb{S}^{d}\right)} .
\end{aligned}
$$

Then the claim follows by applying Hölder's inequality, using Proposition 4.1 to estimate $\left\|\boldsymbol{M}-\boldsymbol{M}_{N}\right\|_{L^{\infty}\left(D ; \mathbb{S}^{d}\right)}$ and Lemma 4.3 to estimate the remaining terms.

Corollary 4.1 For any $q \in[1, \infty)$ and any $0<s<\bar{s}=\tau^{-1}-1 / 2$,

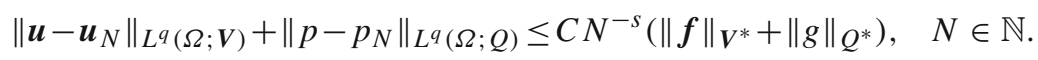

\section{Mixed finite element methods}

We consider a mixed finite element approximation to the deterministic truncated Brinkman problem (4.17). The analysis presented in [15] is augmented to cover the 
case of a nondiagonal permeability matrix $\boldsymbol{M}_{N}$. Let $\mathcal{T}_{h}$ be a quasiuniform and shaperegular partition of $D$ into simplices. The diameter of an element $T \in \mathcal{T}_{h}$ is denoted by $h_{T}$, and the global mesh width $h$ is defined as $h=\max _{T \in \mathcal{T}_{h}} h_{T}$.

We choose the finite element spaces $\boldsymbol{V}_{h} \times Q_{h} \subset \boldsymbol{V} \times Q$ for the mixed finite element discretization of (4.17) as

$$
\begin{aligned}
& \boldsymbol{V}_{h}=\left\{\boldsymbol{v} \in \boldsymbol{V} \cap[C(D)]^{d} ;\left.\boldsymbol{v}\right|_{T} \in\left[P_{k}(T)\right]^{d} \quad \forall T \in \mathcal{T}_{h}\right\}, \\
& Q_{h}=\left\{q \in L_{*}^{2}(D) \cap C(D) ;\left.q\right|_{T} \in P_{k}(T) \quad \forall T \in \mathcal{T}_{h}\right\},
\end{aligned}
$$

in which $P_{k}(T)$ are polynomials of degree $k$. Since the equal order polynomial spaces are not stable for the problem, we employ a mesh-dependent stabilization procedure.

The stabilized finite element formulation of (4.17) reads: Find $\left(\boldsymbol{u}_{N, h}, p_{N, h}\right) \in$ $V_{h} \times Q_{h}$ such that

$$
\mathcal{B}_{N, h}\left(\boldsymbol{u}_{N, h}, p_{N, h} ; \boldsymbol{v}, q\right)=\mathcal{L}_{h}(\boldsymbol{v}, q) \quad \forall(\boldsymbol{v}, q) \in \boldsymbol{V}_{h} \times Q_{h}
$$

The mesh-dependent bilinear form is defined with the stability parameter $\gamma>0$ as

$$
\begin{aligned}
& \mathcal{B}_{N, h}(\boldsymbol{u}, p ; \boldsymbol{v}, q):=\mathcal{B}_{N}(\boldsymbol{u}, p ; \boldsymbol{v}, q) \\
& \left.\quad-\gamma \sum_{T \in \mathcal{T}_{h}} \frac{h_{T}^{2}}{t^{2}+h_{T}^{2}}\left(t^{2} \boldsymbol{M}_{N}^{-1} \boldsymbol{A} \boldsymbol{u}-\boldsymbol{u}-\boldsymbol{M}_{N}^{-1} \nabla p\right), t^{2} \boldsymbol{M}_{N}^{-1} \boldsymbol{A} \boldsymbol{v}-\boldsymbol{v}-\boldsymbol{M}_{N}^{-1} \nabla q\right)_{K}
\end{aligned}
$$

and the corresponding load functional is

$$
\mathcal{L}_{N, h}(\boldsymbol{v}, q):=\mathcal{L}(\boldsymbol{v}, q)+\gamma \sum_{T \in \mathcal{T}_{h}} \frac{h_{T}^{2}}{t^{2}+h_{T}^{2}}\left(\boldsymbol{M}_{N}^{-1} \boldsymbol{f}, t^{2} \boldsymbol{M}_{N}^{-1} \boldsymbol{A} \boldsymbol{v}-\boldsymbol{v}-\boldsymbol{M}_{N}^{-1} \nabla q\right)_{K}
$$

Assuming

$$
-t^{2} \boldsymbol{A} \boldsymbol{u}+\boldsymbol{M}_{N} \boldsymbol{u}+\nabla p=\boldsymbol{f} \in\left[L^{2}(D)\right]^{d},
$$

we have a consistent method, that is

$$
\mathcal{B}_{N, h}\left(\boldsymbol{u}_{N}-\boldsymbol{u}_{N, h}, p_{N}-p_{N, h} ; \boldsymbol{v}, q\right)=0 \quad \forall(\boldsymbol{v}, q) \in \boldsymbol{V}_{h} \times Q_{h}
$$




\subsection{Stability}

To show the stability of the method, we follow [15] and define the following meshdependent norm for the pressure,

$$
\|q\|_{t, h}^{2}:=\sum_{T \in \mathcal{T}_{h}} \frac{h_{T}^{2}}{t^{2}+h_{T}^{2}}\|\nabla q\|_{0, T}^{2}
$$

We first recall the following inverse estimate:

$$
h_{T}^{2}\|\boldsymbol{A} \boldsymbol{v}\|_{0, T}^{2} \leq C_{I}\|\nabla \boldsymbol{v}\|_{0, T}^{2} \quad \forall \boldsymbol{v} \in \boldsymbol{V}_{h} .
$$

Let $\mu_{\min , N}$ and $k$ be defined as in Lemma 4.3. Then we have

Theorem 5.1 Choose $\gamma:=\min \left\{\frac{\mu_{\min , N}}{4}, \frac{k \mu_{\min , N}^{2}}{4 C_{I}}\right\}$. Then for each $N$ there exists $a$ constant $\beta_{N}$ such that

$$
\sup _{(\boldsymbol{v}, q) \in \boldsymbol{V}_{h} \times Q_{h}} \frac{\mathcal{B}_{N, h}(\boldsymbol{w}, r ; \boldsymbol{v}, q)}{\|\boldsymbol{v}\|_{t}+\|q\|_{t, h}} \geq \beta_{N}\left(\|\boldsymbol{w}\|_{t}+\|r\|_{t, h}\right) \quad \forall(\boldsymbol{w}, r) \in \boldsymbol{V}_{h} \times Q_{h} .
$$

Proof Let $(\boldsymbol{w}, r) \in \boldsymbol{V}_{h} \times Q_{h}$ be arbitrary. Then we have

$$
\begin{aligned}
\mathcal{B}_{N, h}(\boldsymbol{w}, r ; \boldsymbol{w},-r)= & t^{2}(\boldsymbol{\epsilon}(\boldsymbol{w}), \boldsymbol{\epsilon}(\boldsymbol{w}))+\left(\boldsymbol{M}_{N} \boldsymbol{w}, \boldsymbol{w}\right) \\
& -\gamma \sum_{T \in \mathcal{T}_{h}} \frac{h_{T}^{2}}{h_{T}^{2}+t^{2}}\left(\left\|t^{2} \boldsymbol{M}_{N}^{-1} \boldsymbol{A} \boldsymbol{w}-\boldsymbol{w}\right\|_{0, T}^{2}-\left\|\boldsymbol{M}_{N}^{-1} \nabla q\right\|_{0, T}^{2}\right) .
\end{aligned}
$$

Estimating the negative terms from above and using the inequality (5.9), we get

$$
\begin{aligned}
\mathcal{B}_{N, h}(\boldsymbol{w}, r ; \boldsymbol{w},-r) \geq & k t^{2}\|\nabla \boldsymbol{w}\|_{0}^{2}+\mu_{\min , N}\|\boldsymbol{w}\|_{0}^{2}+\gamma \mu_{\max , N}^{-2}\|q\|_{t, h}^{2} \\
& -2 \gamma \sum_{T \in \mathcal{T}_{h}} \frac{h_{T}^{2}}{h_{T}^{2}+t^{2}}\left(\mu_{\min , N}^{-2}\left\|t^{2} \boldsymbol{A} \boldsymbol{w}\right\|_{0, T}^{2}+\|\boldsymbol{w}\|_{0, T}^{2}\right) \\
\geq & \left(k-2 \gamma C_{I} \mu_{\min , N}^{-2}\right)\|\nabla \boldsymbol{w}\|_{0}^{2}+\left(\mu_{\min , N}-2 \gamma\right)\|\boldsymbol{w}\|_{0}^{2} \\
& +\gamma \mu_{\max , N}^{-2}\|q\|_{t, h}^{2} \\
\geq & \frac{k}{2}\|\nabla \boldsymbol{w}\|_{0}^{2}+\frac{\mu_{\min , N}}{2}\|\boldsymbol{w}\|_{0}^{2}+\gamma \mu_{\max , N}^{-2}\|q\|_{t, h}^{2} .
\end{aligned}
$$

Thus the theorem holds with

$$
\beta_{N}=\min \left\{\frac{k}{2}, \frac{\mu_{\min , N}}{2}, \frac{\mu_{\min , N}}{4 \mu_{\max , N}^{2}}, \frac{k \mu_{\min , N}^{2}}{4 C_{I} \mu_{\max , N}^{2}}\right\}
$$


Remark 5.1 Note that for first order elements $\boldsymbol{A} \boldsymbol{w}=0$ for every $\boldsymbol{w} \in \boldsymbol{V}_{h}$. Thus it suffices to choose $\gamma:=\frac{\mu_{\min , N}}{4}$ and $\beta_{N}$ will not depend on the constant $C_{I}$ of the inequality (5.9). The smallest eigenvalue can be easily estimated from the stochastic coefficients of the Karhunen-Loève expansion (4.4) for each sample.

Remark 5.2 It is also possible to choose the stabilization parameter elementwise with $\gamma_{T}:=\min \left\{\frac{1}{4} \mu_{\min , N, T}, \frac{k}{4 C_{I}} \mu_{\min , N, T}{ }^{2}\right\}$. Then elementwise estimation gives the sharper lower bound

$$
\beta_{N}=\min _{T \in \mathcal{T}_{h}}\left\{\frac{k}{2}, \frac{\mu_{\min , N}}{2}, \frac{\mu_{\min , N, T}}{4 \mu_{\max , N, T^{2}}}, \frac{k \mu_{\min , N, T}}{4 C_{I} \mu_{\max , N, T^{2}}}\right\} .
$$

Next, we use the result for the mesh-dependent norm to show that the stability holds also in the continuous pressure norm. With a small modification of the arguments presented in [12], one has

Lemma 5.1 There exists constants $C_{1}$ and $C_{2}$ independent of the mesh width $h$, and the parameters $t$ and $\boldsymbol{M}_{N}$ such that

$$
\sup _{\boldsymbol{w} \in \boldsymbol{V}_{h}} \frac{b(\boldsymbol{w}, q)}{\|\boldsymbol{w}\|_{t}} \geq C_{1}\|q\|_{t}-C_{2}\|q\|_{t, h} .
$$

Now we are ready to prove the stability in the continuous norm.

Theorem 5.2 Suppose the stability of Theorem 5.1 holds. Then for each $N$ there exists a constant $\widehat{\beta_{N}}$ such that

$$
\sup _{(\boldsymbol{v}, q) \in \boldsymbol{V}_{h} \times Q_{h}} \frac{\mathcal{B}_{N, h}(\boldsymbol{w}, r ; \boldsymbol{v}, q)}{\|\boldsymbol{v}\|_{t}+\|q\|_{t, h}} \geq \widehat{\beta_{N}}\left(\|\boldsymbol{w}\|_{t}+\|r\|_{t}\right) \quad \forall(\boldsymbol{w}, r) \in \boldsymbol{V}_{h} \times Q_{h} .
$$

Furthermore, the constant $\widehat{\beta_{N}}$ has a polynomial dependence on the constant $\beta_{N}$ and the constants $\alpha_{N}$ and $c_{a, N}$.

Proof Let $\boldsymbol{w} \in \boldsymbol{V}_{h}$ be a function for which the supremum is attained in Lemma 5.1, and assume that $\boldsymbol{w}$ is scaled such that $\|\boldsymbol{w}\|_{t}=\|q\|_{t, h}$. Employing the techniques of the proof in [12, Lemma 3.2] it can be shown that for every $(\boldsymbol{v}, q) \in \boldsymbol{V}_{h} \times Q_{h}$ we have

$$
\mathcal{B}_{N, h}(\boldsymbol{v}, q ;-\boldsymbol{w}, 0) \geq-C_{3}\|\boldsymbol{v}\|_{t}^{2}+\left(C_{4}\|q\|_{t}-C_{5}\|q\|_{t, h}\right)\|q\|_{t, h},
$$

in which the constants $C_{3}, C_{4}$, and $C_{5}$ are polynomial functions of the constants $\alpha_{N}$ and $c_{a, N}$. Now, choosing $0<\delta=\frac{\beta_{N}}{2\left(C_{3}+C_{5}\right)}$ yields

$$
\begin{aligned}
\mathcal{B}_{N, h}(\boldsymbol{v}, q ; \boldsymbol{v}-\delta \boldsymbol{w},-q) & \geq\left(\beta_{N}-\delta C_{3}\right)\|\boldsymbol{v}\|_{t}^{2}+\delta C_{4}\|q\|_{t}\|q\|_{t, h}+\left(\beta_{N}-\delta C_{5}\right)\|q\|_{t, h}^{2} \\
& \geq \frac{\beta_{N}}{2}\|\boldsymbol{v}\|_{t}^{2}+\frac{\beta_{N} C_{4}}{2\left(C_{3}+C_{5}\right)}\|q\|_{t}\|q\|_{t, h} .
\end{aligned}
$$




\subsection{A priori estimates}

We approximate the error of the velocity field in the $\|\cdot\|_{t}$-norm and the error of the pressure field in both the continuous norm $\|\cdot\|_{t}$ and in the computable meshdependent norm $\|\cdot\|_{t, h}$. The following quasi-optimal error bound holds. Note that a term estimating the residual is included, as is typical for stabilized methods.

Theorem 5.3 Let the stabilization parameter $\gamma$ be chosen as in Theorem 5.1. Then the finite element solution $\left(\boldsymbol{u}_{N, h}, p_{N, h}\right)$ of (4.17) is $\mathbb{P}$-measurable. Moreover, for every $N \in \mathbb{N}$ and a constant $C$ independent of $\omega$, the mesh width $h$ and of the parameters $t$ and $\boldsymbol{M}_{N}$, with the random variables $X=\alpha, X=c_{a}, X=\alpha_{N}^{-1}$ and $X=c_{a, N}$ satisfying (4.20),

$$
\begin{aligned}
& \left\|\boldsymbol{u}_{N}-\boldsymbol{u}_{N, h}\right\|_{t}+\left\|p_{N}-p_{N, h}\right\|_{t, h} \\
& \leq \frac{C}{\beta_{N}}\left(2+c_{a, N}+\frac{1+\alpha_{N}}{\alpha_{N}^{2}}\right) \inf _{\substack{(\boldsymbol{v}, q) \epsilon \\
V_{h} \times Q_{h}}}\left\{\left\|p_{N}-q\right\|_{t}+\left\|p_{N}-q\right\|_{t, h}+\left\|\boldsymbol{u}_{N}-\boldsymbol{v}\right\|_{t}\right. \\
& \left.\quad+\left(\sum_{T \in \mathcal{T}_{h}} \frac{t^{2}}{h_{T}^{2}}\left\|\boldsymbol{u}_{N}-\boldsymbol{v}\right\|_{0, T}^{2}+\frac{h_{T}^{2}}{h_{T}^{2}+t^{2}}\left\|t^{2} \boldsymbol{A} \boldsymbol{v}-\boldsymbol{M}_{N} \boldsymbol{v}-\nabla q+\boldsymbol{f}\right\|_{0, T}^{2}\right)^{\frac{1}{2}}\right\} .
\end{aligned}
$$

Proof Using the triangle inequality we have for arbitrary $(\boldsymbol{v}, q) \in \boldsymbol{V}_{h} \times Q_{h}$

$$
\begin{aligned}
& \left\|\boldsymbol{u}_{N}-\boldsymbol{u}_{N, h}\right\|_{t}+\left\|p_{N}-p_{N, h}\right\|_{t, h} \\
& \quad \leq\left\|\boldsymbol{u}_{N}-\boldsymbol{v}\right\|_{t}+\left\|p_{N}-q\right\|_{t, h}+\left\|\boldsymbol{v}-\boldsymbol{u}_{N, h}\right\|_{t}+\left\|q-p_{N, h}\right\|_{t, h} .
\end{aligned}
$$

Using Theorem 5.1 we have functions $(\boldsymbol{w}, r) \in \boldsymbol{V}_{h} \times Q_{h}$ with

$$
\|\boldsymbol{w}\|_{t}+\|r\|_{t, h} \leq C
$$

such that there holds

$$
\begin{aligned}
\beta_{N}\left\|\boldsymbol{v}-\boldsymbol{u}_{N, h}\right\|_{t}+\left\|q-p_{N, h}\right\|_{t, h} & \leq \mathcal{B}_{N, h}\left(\boldsymbol{u}_{N, h}-\boldsymbol{v}, p_{N, h}-q ; \boldsymbol{w}, r\right) \\
& =\mathcal{B}_{N, h}\left(\boldsymbol{u}_{N}-\boldsymbol{v}, p_{N}-q ; \boldsymbol{w}, r\right) \\
& =\mathcal{B}_{N}\left(\boldsymbol{u}_{N}-\boldsymbol{v}, p_{N}-q ; \boldsymbol{w}, r\right)-S,
\end{aligned}
$$

in which by the assumption (5.6)

$$
\begin{aligned}
S= & \gamma \sum_{T \in \mathcal{T}_{h}} \frac{h_{T}^{2}}{h_{T}^{2}+t^{2}}\left(-t^{2} \boldsymbol{M}_{N}^{-1} \boldsymbol{A} \boldsymbol{v}+\boldsymbol{v}+\boldsymbol{M}_{N}^{-1} \nabla q-\boldsymbol{M}_{N}^{-1} \boldsymbol{f},\right. \\
& \left.t^{2} \boldsymbol{M}_{N}^{-1} \boldsymbol{A} \boldsymbol{w}-\boldsymbol{w}-\boldsymbol{M}_{N}^{-1} \nabla r\right)_{K} .
\end{aligned}
$$


Estimating the first term yields

$$
\begin{aligned}
\mathcal{B}_{N}\left(\boldsymbol{u}_{N}-\boldsymbol{v}, p_{N}-q ; \boldsymbol{w}, r\right) \\
\quad \leq c_{a, N}\left\|\boldsymbol{u}_{N}-\boldsymbol{v}\right\|_{t}\|\boldsymbol{w}\|_{t}-b\left(\boldsymbol{w}, p_{N}-q\right)+\left(\boldsymbol{u}_{N}-\boldsymbol{v}, \nabla r\right) \\
\leq c_{a, N}\left\|\boldsymbol{u}_{N}-\boldsymbol{v}\right\|_{t}\|\boldsymbol{w}\|_{t}+\left\|p_{N}-q\right\|_{t}\|\boldsymbol{w}\|_{t} \\
\quad+\left(\sum_{T \in \mathcal{T}_{h}} \frac{h_{T}^{2}}{h_{T}^{2}+t^{2}}\left\|\boldsymbol{u}_{N}-\boldsymbol{v}\right\|_{0, T}^{2}\right)^{1 / 2}\left(\sum_{T \in \mathcal{T}_{h}} \frac{h_{T}^{2}}{h_{T}^{2}+t^{2}}\|\nabla r\|_{0, T}^{2}\right)^{1 / 2} \\
\quad \leq\left(\left(1+c_{a, N}\right)\left\|\boldsymbol{u}_{N}-\boldsymbol{v}\right\|_{t}+\left\|p_{N}-q\right\|_{t}+t\left(\sum_{T \in \mathcal{T}_{h}} h_{T}^{-2}\left\|\boldsymbol{u}_{N}-\boldsymbol{v}\right\|_{0, T}^{2}\right)^{1 / 2}\right) \\
\quad \times\left(\|\boldsymbol{w}\|_{t}+\|r\|_{t, h}\right) .
\end{aligned}
$$

Turning to the stabilizing term, we have using the inverse inequality (5.9)

$$
\begin{aligned}
S \leq & \left(\sum_{T \in \mathcal{T}_{h}} \frac{1}{\mu_{\min , N}^{2}} \frac{h_{T}^{2}}{h_{T}^{2}+t^{2}}\left\|-t^{2} \boldsymbol{A} \boldsymbol{v}+\boldsymbol{M}_{N} \boldsymbol{v}+\nabla q-\boldsymbol{f}\right\|_{0, T}^{2}\right)^{\frac{1}{2}} \\
& \times\left(\sum_{T \in \mathcal{T}_{h}} \frac{h_{T}^{2}}{h_{T}^{2}+t^{2}}\left\|t^{2} \boldsymbol{M}_{N}^{-1} \boldsymbol{A} \boldsymbol{w}-\boldsymbol{w}-\boldsymbol{M}_{N}^{-1} \nabla r\right\|_{0, T}^{2}\right)^{\frac{1}{2}} \\
\leq & C \frac{1+\mu_{\min , N}}{\mu_{\min , N}^{2}}\left(\sum_{T \in \mathcal{T}_{h}} \frac{h_{T}^{2}}{h_{T}^{2}+t^{2}}\left\|t^{2} \boldsymbol{A} \boldsymbol{v}-\boldsymbol{M}_{N} \boldsymbol{v}-\nabla q+\boldsymbol{f}\right\|_{0, T}^{2}\right)^{\frac{1}{2}} \\
& \times\left(\|\boldsymbol{w}\|_{t}+\|r\|_{t, h}\right) .
\end{aligned}
$$

With exactly the same arguments as above, and using Lemma 5.2, we can derive a quasioptimal a priori result in the continuous pressure norm with a stability constant $\widehat{\beta_{N}}$ instead of $\beta_{N}$.

Theorem 5.4 Let the assumption of Theorem 5.3 hold. Then we have

$$
\begin{aligned}
& \left\|\boldsymbol{u}_{N}-\boldsymbol{u}_{N, h}\right\|_{t}+\left\|p_{N}-p_{N, h}\right\|_{t} \\
& \leq \frac{C}{\widehat{\beta_{N}}}\left(2+c_{a, N}+\frac{1+\alpha_{N}}{\alpha_{N}^{2}}\right) \inf _{\substack{(\boldsymbol{v}, q) \in \\
V_{h} \times Q_{h}}}\left\{\left\|p_{N}-q\right\|_{t}+\left\|\boldsymbol{u}_{N}-\boldsymbol{v}\right\|_{t}\right. \\
& \left.\quad+\left(\sum_{T \in \mathcal{T}_{h}} \frac{t^{2}}{h_{T}^{2}}\left\|\boldsymbol{u}_{N}-\boldsymbol{v}\right\|_{0, T}^{2}+\frac{h_{T}^{2}}{h_{T}^{2}+t^{2}}\left\|t^{2} \boldsymbol{A} \boldsymbol{v}-\boldsymbol{M}_{N} \boldsymbol{v}-\nabla q+\boldsymbol{f}\right\|_{0, T}^{2}\right)^{\frac{1}{2}}\right\} .
\end{aligned}
$$




\subsection{A priori estimate for a regular solution}

Even though in practice the solution to the equations is seldom smooth, it is often beneficial to write the error estimate assuming a smooth solution and thus exposing the convergence rates. Assuming $\boldsymbol{u}_{N} \in\left[H^{k+1}(D)\right]^{d}$ and $p_{N} \in H^{k+1}(D)$ we have the following convergence result for a polynomial approximation of degree $k$.

Theorem 5.5 There is a constant $C$ independent of the mesh width $h$ and of the parameters $t$ and $\boldsymbol{M}_{N}$, such that in the continuous pressure norm with random variables $X=\alpha, X=c_{a}, X=\alpha_{N}^{-1}$ and $X=c_{a, N}$ as in (4.20) independent of $h$ and $0 \leq t \leq 1$,

$$
\begin{aligned}
& \left\|\boldsymbol{u}_{N}-\boldsymbol{u}_{N, h}\right\|_{t}+\left\|p_{N}-p_{N, h}\right\|_{t} \\
& \quad \leq C R\left(\alpha_{N}, c_{a, N}\right)\left[(t+h) h^{k}\left\|\boldsymbol{u}_{N}\right\|_{k+1}+(t+h)^{-1} h^{k+1}\left\|p_{N}\right\|_{k+1}\right],
\end{aligned}
$$

in which $R(\cdot, \cdot)$ is a rational function of the two arguments. The same result holds also for the mesh-dependent pressure norm.

Remark 5.3 In the limit $t=0$ the method constitutes a stabilized mixed finite element approximation of the Darcy equation. Also the norms (2.5) and (5.8) reduce to those used in the dual mixed formulation of the Darcy problem. However, the method is not optimal since we only get order $k$ convergence in the aforementioned norms, as opposed to the $k+1$ convergence provided by the $H$ (div)-conforming elements, cf. $[17,19]$.

\section{The multi-level Monte Carlo method}

\subsection{Single level Monte Carlo}

We have shown in Proposition 3.2 and in Sect. 4 that the solutions $U:=(\boldsymbol{u}, p)$ and $U_{N}:=\left(\boldsymbol{u}_{N}, p_{N}\right)$ of the stochastic Brinkman problem with lognormal permeability $\boldsymbol{M}$ from (3.1) and $\boldsymbol{M}_{N}$ from (4.10), respectively, admit bounded moments of any finite order $q$. Choosing $q=2 m$ for $m \in \mathbb{N}$, this implies that the second moments of the $m$-point correlation functions of the random fields $U$ and $U_{N}$ are bounded and can be estimated by Monte Carlo (MC) sampling of the mixed FE approximations of the random solutions.

We give an a priori error analysis of the resulting MC-MFEM. For the sake of brevity, we develop the results only for $m=1$ but hasten to add that all results which follow admit generalizations to moments of order $m>1$; we refer to [4] for statements and proofs in the case of Darcy equations with random permeability.

Let us first address the Single Level MC method. Here, for a given, fixed truncation order $N$ in (4.10), and for a given pair $\boldsymbol{V}_{h} \times Q_{h}$ of FE spaces, we draw $M \geq 1$ independent, identically distributed realizations $\hat{\boldsymbol{M}}_{i}(x):=\boldsymbol{M}_{N}\left(x, \omega_{i}\right), i=1, \ldots, M$, using the $N$-term truncated Karhunen-Loève expansion (4.10). Note that the work to do so grows as $O(M N)$ as $N, M \rightarrow \infty$. For each $\hat{\boldsymbol{M}}_{i}$, we solve the FE equations (5.3). We assume for now that the FE solutions $\hat{U}_{N, h}^{i}:=\left(\hat{\boldsymbol{u}}_{N, h}^{i}, \hat{p}_{N, h}^{i}\right)$ are computed exactly, 
although in general an approximate solution (obtained, for example, by a multilevel method) will suffice. We denote by $W(h)$ the computational work for the numerical solution of the linear system (5.3).

In the Single Level MC MFEM, we estimate the mathematical expectation $\mathbb{E}[U]$ by the ensemble average of the $M$ MFEM sample solutions, i.e. by

$$
E_{M}\left[U_{N, h}\right]:=\frac{1}{M} \sum_{i=1}^{M} \hat{U}_{N, h}^{i}
$$

The total work for the computation of this estimate is $O(M N W(h))$. For the convergence analysis of this MC-FE approximation, we assume as usual (see e.g. [4]) the FE solutions $\hat{U}_{N, h}^{i}$ corresponding to the $M$ coefficient draws $\hat{\boldsymbol{M}}_{i}$ in (6.1) to be $M$ i.i.d. copies of the random FE solutions $U_{N, h}=\left(\boldsymbol{u}_{N, h}, p_{N, h}\right)$ defined in (5.3). To estimate the MC-MFEM error, we measure the FE error in mean-square in probability, i.e.

$$
\begin{aligned}
& \left\|\mathbb{E}[U]-E_{M}\left[U_{N, h}\right]\right\|_{L^{2}(\Omega, V \times Q)} \\
& =\left(\mathbb{E}\left[\left\|\mathbb{E}[\boldsymbol{u}]-\frac{1}{M} \sum_{i=1}^{M} \hat{\boldsymbol{u}}_{N, h}^{i}\right\|_{t}^{2}+\left\|\mathbb{E}[p]-\frac{1}{M} \sum_{i=1}^{M} \hat{p}_{N, h}^{i}\right\|_{t}^{2}\right]\right)^{1 / 2} .
\end{aligned}
$$

Combining the a priori error bound of Theorem 5.5 with Lemma 4.3 and a standard argument as in Lemma 4.1 of [4], we obtain the following convergence property.

Theorem 6.1 For any $q>1,0<s<\tau^{-1}-1 / 2$ and $0 \leq t<\infty$, if the solution to the truncated problem (4.17) satisfies

$$
\boldsymbol{u}_{N} \in L^{q}\left(\Omega ; H^{k+1}(D)^{d}\right) \text { and } p_{N} \in L^{q}\left(\Omega ; H_{*}^{k+1}(D)\right)
$$

for all $N$, then there is a constant $C$ independent of $N, h, M$ and $t$ such that

$$
\begin{aligned}
& \left\|\mathbb{E}[U]-E_{M}\left[U_{N, h}\right]\right\|_{L^{2}(\Omega, \boldsymbol{V} \times Q)} \leq C\left(\left(N^{-s}\left(\|\boldsymbol{f}\|_{\boldsymbol{V}^{*}}+\|g\|_{Q^{*}}\right)\right.\right. \\
& \left.+(t+h) h^{k}\left\|\boldsymbol{u}_{N}\right\|_{L^{q}\left(\Omega ; H^{k+1}(D)^{d}\right)}+(t+h)^{-1} h^{k+1}\left\|p_{N}\right\|_{L^{q}\left(\Omega ; H^{k+1}(D)\right)}\right)^{2} \\
& +M^{-1}\left(\|\boldsymbol{f}\|_{V^{*}}+\|g\|_{\left.Q^{*}\right)^{2}}\right)^{1 / 2}
\end{aligned}
$$

where $k$ is the order of the mixed finite element discretization. The total work for the computation of $E_{M}\left[U_{N, h}\right]$ is $O\left(M\left(N h^{-d}+W(h)\right)\right)$.

Proof Since $\mathbb{E}\left[E_{M}\left[U_{N, h}\right]\right]=\mathbb{E}\left[U_{N, h}\right]$, we can expand the error as

$$
\begin{aligned}
& \left\|\mathbb{E}[U]-E_{M}\left[U_{N, h}\right]\right\|_{L^{2}(\Omega, \boldsymbol{V} \times Q)}^{2} \\
& \quad=\left\|\mathbb{E}[U]-\mathbb{E}\left[U_{N, h}\right]\right\|_{\boldsymbol{V} \times Q}^{2}+\left\|\mathbb{E}\left[U_{N, h}\right]-E_{M}\left[U_{N, h}\right]\right\|_{L^{2}(\Omega, \boldsymbol{V} \times Q)}^{2} .
\end{aligned}
$$


By the a priori error bound of Theorem 5.5, the truncation error estimate Corollary 4.1 and the bound Lemma 4.3, the first term satisfies

$$
\begin{aligned}
\left\|\mathbb{E}[U]-\mathbb{E}\left[U_{N, h}\right]\right\|_{\boldsymbol{V} \times Q} \leq & \left\|U-U_{N, h}\right\|_{L^{1}(\Omega ; \boldsymbol{V} \times Q)} \\
\leq & N^{-s}\left(\|\boldsymbol{f}\|_{V^{*}}+\|g\|_{Q^{*}}\right)+(t+h) h^{k}\left\|\boldsymbol{u}_{N}\right\|_{L^{q}\left(\Omega ; H^{k+1}(D)^{d}\right)} \\
& +(t+h)^{-1} h^{k+1}\left\|p_{N}\right\|_{L^{q}\left(\Omega ; H^{k+1}(D)\right)} .
\end{aligned}
$$

For the second term, using that the samples $\hat{U}_{N, h}^{i}$ are independent and have expectation $\mathbb{E}\left[U_{N, h}\right]$, we have

$$
\begin{aligned}
\left\|\mathbb{E}\left[U_{N, h}\right]-E_{M}\left[U_{N, h}\right]\right\|_{L^{2}(\Omega, \boldsymbol{V} \times Q)}^{2} & =\mathbb{E}\left[\left\|\mathbb{E}\left[U_{N, h}\right]-\frac{1}{M} \sum_{i=1}^{M} \hat{U}_{N, h}^{i}\right\|_{\boldsymbol{V} \times Q}^{2}\right] \\
& =\mathbb{E}\left[\left\|\frac{1}{M} \sum_{i=1}^{M} \mathbb{E}\left[U_{N, h}\right]-\hat{U}_{N, h}^{i}\right\|_{\boldsymbol{V} \times Q}^{2}\right] \\
& =\frac{1}{M} \mathbb{E}\left[\left\|U_{N, h}-\mathbb{E}\left[U_{N, h}\right]\right\|_{\boldsymbol{V} \times Q}^{2}\right],
\end{aligned}
$$

and using Lemma 4.3, due to the stability of the mixed finite element discretization,

$$
\mathbb{E}\left[\left\|U_{N, h}-\mathbb{E}\left[U_{N, h}\right]\right\|_{\boldsymbol{V} \times Q}^{2}\right] \leq \mathbb{E}\left[\left\|U_{N, h}\right\|_{\boldsymbol{V} \times Q}^{2}\right] \leq C\left(\|\boldsymbol{f}\|_{\boldsymbol{V}^{*}}+\|g\|_{Q^{*}}\right)^{2}
$$

The computational cost is $O\left(N h^{-d}\right)$ for the summation of the Karhunen-Loève series, and $W(h)$ for the solution of the resulting linear system; both operations are performed $M$ times, resulting in a total cost of $O\left(M\left(N h^{-d}+W(h)\right)\right)$.

We remark that due to the potentially low regularity of the Gaussian field $\boldsymbol{M}(x, \omega)$, in general the regularity of the solution $U$ is rather low, and we can realistically expect only $0<k \leq 1$ in (6.3) resp. in (6.4) for $U$ in place of $U_{N}$. Due to the truncation of the Gaussian field, the regularity of $U_{N}$ is higher, although Sobolev norms of $\boldsymbol{u}_{N}$ and $p_{N}$ may not be bounded uniformly in $N$.

\subsection{Multi-level Monte Carlo}

A substantial efficiency increase compared to SLMC-MFEM can be obtained by the Multi-Level Monte Carlo Mixed Finite Element Method (MLMC-MFEM), which is based on a hierarchic family of FE discretizations.

Let $\left\{\mathcal{T}_{l}\right\}_{l=0}^{L}$ denote a sequence of regular, simplicial meshes with mesh widths $h_{l}:=$ $\max \left\{\right.$ diam $\left.T: T \in \mathcal{T}_{l}\right\}$. For example, $\mathcal{T}_{l}$ could be obtained by $l$-fold regular subdivision of an initial regular simplicial triangulation $\mathcal{T}_{0}$ of $D$, in which case $h_{l}=2^{-l} h_{0}$. To ease notation, we denote the corresponding FE spaces $\boldsymbol{V}_{h_{l}} \times Q_{h_{l}}$ by $\boldsymbol{V}_{l} \times Q_{l}$ in the following. 
We also introduce a sequence $\left(N_{l}\right)_{l=0}^{L}$ of truncations of the series expansion (4.4). The FE solution of the Brinkman problem (5.3) on the mesh $\mathcal{T}_{l}$ with lognormal permeability $\boldsymbol{M}_{N_{l}}$ is denoted by $U_{l}:=\left(\boldsymbol{u}_{l}, p_{l}\right)$, with $\boldsymbol{u}_{l}:=\boldsymbol{u}_{N_{l}, h_{l}}$ and $p_{l}:=p_{N_{l}, h_{l}}$. With the convention $U_{-1}:=0$, we may write

$$
U_{L}=\sum_{l=0}^{L} U_{l}-U_{l-1}
$$

By linearity of the mathematical expectation, it follows that

$$
\mathbb{E}\left[U_{L}\right]=\sum_{l=0}^{L} \mathbb{E}\left[U_{l}-U_{l-1}\right]
$$

We replace each expectation in this telescoping sum by an MC estimate, with a leveldependent number $M_{l}$ of samples. This yields the MLMC-MFE estimator

$$
E^{L}[U]:=\sum_{l=0}^{L} E_{M_{l}}\left[U_{l}-U_{l-1}\right]
$$

We note that, since $U_{-1}=0, E^{L}[U]$ is an unbiased estimator for $\mathbb{E}\left[U_{L}\right]$,

$$
\mathbb{E}\left[E^{L}[U]\right]=\sum_{l=0}^{L} \mathbb{E}\left[E_{M_{l}}\left[U_{l}-U_{l-1}\right]\right]=\sum_{l=0}^{L} \mathbb{E}\left[U_{l}-U_{l-1}\right]=\mathbb{E}\left[U_{L}\right]
$$

Consequently, $\mathbb{E}\left[\mathbb{E}\left[U_{L}\right]-E^{L}[U]\right]=0$, and since $\mathbb{E}[U]-\mathbb{E}\left[U_{L}\right]$ is a deterministic quantity,

$$
\begin{aligned}
\left\|\mathbb{E}[U]-E^{L}[U]\right\|_{L^{2}(\Omega ; \boldsymbol{V} \times Q)}^{2}= & \left\|\mathbb{E}[U]-\mathbb{E}\left[U_{L}\right]\right\|_{\boldsymbol{V} \times Q}^{2} \\
& +\left\|\mathbb{E}\left[U_{L}\right]-E^{L}[U]\right\|_{L^{2}(\Omega ; \boldsymbol{V} \times Q)}^{2} .
\end{aligned}
$$

By definition and due to Theorem 6.1, the computational cost of $E^{L}[U]$ is

$$
W^{L} \sim \sum_{l=0}^{L} M_{l}\left(N_{l} h_{l}^{-d}+W\left(h_{l}\right)\right)
$$

The a priori error bound for the MLMC estimator is as follows.

Theorem 6.2 If $M_{l} \geq C M_{l-1}$ for $l=1, \ldots, L$ then for any $q>2$ and $0<s<$ $\tau^{-1}-1 / 2$, if the solution to the truncated problem (4.17) satisfies

$$
\boldsymbol{u}_{N} \in L^{q}\left(\Omega ; H^{k+1}(D)^{d}\right) \text { and } p_{N} \in L^{q}\left(\Omega ; H_{*}^{k+1}(D)\right)
$$


for all $N$, there is a constant $C$ independent of $\left(N_{l}\right)_{l=0}^{L},\left(h_{l}\right)_{l=0}^{L}$ and $\left(M_{l}\right)_{l=0}^{L}$ such that

$$
\begin{aligned}
& \left\|\mathbb{E}\left[U_{L}\right]-E^{L}[U]\right\|_{L^{2}(\Omega ; \boldsymbol{V} \times Q)} \\
& \leq C\left(\frac{1}{M_{0}}\left(\|\boldsymbol{f}\|_{\boldsymbol{V}^{*}}+\|g\|_{Q^{*}}\right)^{2}+\sum_{l=1}^{L} \frac{1}{M_{l}}\left(N_{l}^{-s}\left(\|\boldsymbol{f}\|_{\boldsymbol{V}^{*}}+\|g\|_{Q^{*}}\right)\right.\right. \\
& \left.\left.\quad+\left(t+h_{l}\right) h_{l}^{k}\left\|\boldsymbol{u}_{N_{l}}\right\|_{L^{q}\left(\Omega ; H^{k+1}(D)^{d}\right)}+\left(t+h_{l}\right)^{-1} h_{l}^{k+1}\left\|p_{N_{l}}\right\|_{L^{q}\left(\Omega ; H_{*}^{k+1}(D)\right)}\right)^{2}\right)^{1 / 2}
\end{aligned}
$$

and

$$
\begin{aligned}
& \left\|\mathbb{E}[U]-\mathbb{E}\left[U_{L}\right]\right\|_{\boldsymbol{V} \times Q} \\
& \leq C\left(N_{L}^{-s}\left(\|\boldsymbol{f}\|_{\boldsymbol{V}^{*}}+\|g\|_{Q^{*}}\right)+\left(t+h_{L}\right) h_{L}^{k}\left\|\boldsymbol{u}_{N_{L}}\right\|_{L^{q}\left(\Omega ; H^{k+1}(D)^{d}\right)}\right. \\
& \left.\quad+\left(t+h_{L}\right)^{-1} h_{L}^{k+1}\left\|p_{N_{L}}\right\|_{L^{q}\left(\Omega ; H_{*}^{k+1}(D)\right)}\right),
\end{aligned}
$$

where $k$ is the order of the mixed finite element discretization.

Proof Using independence of the samples in the MC estimators on all levels, we have

$$
\begin{aligned}
\left\|\mathbb{E}\left[U_{L}\right]-E^{L}[U]\right\|_{L^{2}(\Omega ; \boldsymbol{V} \times Q)}^{2} & \\
= & \mathbb{E}\left[\left\|\mathbb{E}\left[U_{L}\right]-\sum_{l=0}^{L} \frac{1}{M_{l}} \sum_{i=1}^{M_{l}} \hat{U}_{l}^{i}-\hat{U}_{l-1}^{i}\right\|_{\boldsymbol{V} \times Q}^{2}\right] \\
= & \mathbb{E}\left[\left\|\sum_{l=0}^{L} \frac{1}{M_{l}} \sum_{i=1}^{M_{l}}\left(\mathbb{E}\left[U_{l}-U_{l-1}\right]-\left(\hat{U}_{l}^{i}-\hat{U}_{l-1}^{i}\right)\right)\right\|_{\boldsymbol{V} \times Q}^{2}\right] \\
= & \sum_{l=0}^{L} \frac{1}{M_{l}} \mathbb{E}\left[\left\|U_{l}-U_{l-1}-\mathbb{E}\left[U_{l}-U_{l-1}\right]\right\|_{\boldsymbol{V} \times Q}^{2}\right] .
\end{aligned}
$$

Since $U_{-1}=0$, the first term in this sum satisfies

$$
\mathbb{E}\left[\left\|U_{0}-\mathbb{E}\left[U_{0}\right]\right\|_{\boldsymbol{V} \times Q}^{2}\right] \leq \mathbb{E}\left[\left\|U_{0}\right\|_{\boldsymbol{V} \times Q}^{2}\right] \leq C\left(\|\boldsymbol{f}\|_{\boldsymbol{V}^{*}}+\|g\|_{Q^{*}}\right)^{2}=: C \boldsymbol{\Xi}^{2}
$$

for any $q>2$ due to Lemma 4.3 and the estimates in Proposition 3.2. For the other terms in the sum, we estimate

$$
\begin{aligned}
\mathbb{E}[ & \left.\left\|U_{l}-U_{l-1}-\mathbb{E}\left[U_{l}-U_{l-1}\right]\right\|_{\boldsymbol{V} \times Q}^{2}\right] \leq \mathbb{E}\left[\left\|U_{l}-U_{l-1}\right\|_{\boldsymbol{V} \times Q}^{2}\right] \\
\leq & \mathbb{E}\left[\left(\left\|U_{N_{l}}-U_{l}\right\|_{\boldsymbol{V} \times Q}+\left\|U_{N_{l}}-U_{N_{l-1}}\right\| \boldsymbol{V} \times Q+\left\|U_{N_{l-1}}-U_{l-1}\right\|_{\boldsymbol{V} \times Q}\right)^{2}\right] \\
\leq & \left(\left\|U_{N_{l}}-U_{l}\right\|_{L^{2}(\Omega ; \boldsymbol{V} \times Q)}+\left\|U_{N_{l}}-U_{N_{l-1}}\right\|_{L^{2}(\Omega ; \boldsymbol{V} \times Q)}\right. \\
& \left.+\left\|U_{N_{l-1}}-U_{l-1}\right\|_{L^{2}(\Omega ; \boldsymbol{V} \times Q)}\right)^{2} .
\end{aligned}
$$


Due to Lemma 4.3 and Theorem 5.5, using Hölder's inequality, we have

$$
\begin{aligned}
& \left\|U_{N_{l}}-U_{l}\right\|_{L^{2}(\Omega ; \boldsymbol{V} \times Q)} \\
& \quad \leq C\left(\left(t+h_{l}\right) h_{l}^{k}\left\|\boldsymbol{u}_{N_{l}}\right\|_{L^{q}\left(\Omega ; H^{k+1}(D)^{d}\right)}+\left(t+h_{l}\right)^{-1} h_{l}^{k+1}\left\|p_{N_{l}}\right\|_{L^{q}\left(\Omega ; H_{*}^{k+1}(D)\right)}\right)
\end{aligned}
$$

for any $q>2$. Furthermore, Corollary 4.1 implies

$$
\begin{aligned}
\left\|U_{N_{l}}-U_{N_{l-1}}\right\|_{L^{2}(\Omega ; \boldsymbol{V} \times Q)} & \leq\left\|U-U_{N_{l}}\right\|_{L^{2}(\Omega ; \boldsymbol{V} \times Q)}+\left\|U-U_{N_{l-1}}\right\|_{L^{2}(\Omega ; \boldsymbol{V} \times Q)} \\
& \leq C\left(N_{l}^{-s}+N_{l-1}^{-s}\right)\left(\|\boldsymbol{f}\|_{\boldsymbol{V}^{*}}+\|g\|_{Q^{*}}\right)
\end{aligned}
$$

for any $0<s<\bar{s}=\tau^{-1}-1 / 2$. We abbreviate

$$
\begin{aligned}
\Theta_{l}:= & N_{l}^{-s}\left(\|\boldsymbol{f}\|_{V^{*}}+\|g\|_{Q^{*}}\right) \\
& +\left(t+h_{l}\right) h_{l}^{k}\left\|\boldsymbol{u}_{N_{l}}\right\|_{L^{q}\left(\Omega ; H^{k+1}(D)^{d}\right)}+\left(t+h_{l}\right)^{-1} h_{l}^{k+1}\left\|p_{N_{l}}\right\|_{L^{q}\left(\Omega ; H_{*}^{k+1}(D)\right)}
\end{aligned}
$$

for $l=0,1, \ldots, L$. Combining the above estimates, we arrive at

$$
\left\|\mathbb{E}\left[U_{L}\right]-E^{L}[U]\right\|_{L^{2}(\Omega ; \boldsymbol{V} \times Q)} \leq C\left(\frac{\Xi^{2}}{M_{0}}+\sum_{l=1}^{L} \frac{1}{M_{l}}\left(\Theta_{l}+\Theta_{l-1}\right)^{2}\right)^{1 / 2}
$$

By the triangle inequality, the right hand side is less than

$$
\left(\frac{\Xi^{2}}{M_{0}}+\sum_{l=1}^{L} \frac{\Theta_{l}^{2}}{M_{l}}\right)^{1 / 2}+\left(\sum_{l=0}^{L-1} \frac{\Theta_{l}^{2}}{M_{l+1}}\right)^{1 / 2}
$$

Since $M_{l} \geq C M_{l-1}$ by assumption, and noting that we may replace $\Theta_{0}$ by $\Xi$ in the above estimate, it follows that the latter term is bounded by the former. Therefore,

$$
\left\|\mathbb{E}\left[U_{L}\right]-E^{L}[U]\right\|_{L^{2}(\Omega ; \boldsymbol{V} \times Q)} \leq C\left(\frac{\Xi^{2}}{M_{0}}+\sum_{l=1}^{L} \frac{\Theta_{l}^{2}}{M_{l}}\right)^{1 / 2} .
$$

Similarly, we estimate

$\left\|\mathbb{E}[U]-\mathbb{E}\left[U_{L}\right]\right\|_{\boldsymbol{V} \times Q} \leq\left\|U-U_{N_{L}}\right\|_{L^{1}(\Omega ; \boldsymbol{V} \times Q)}+\left\|U_{N_{L}}-U_{L}\right\|_{L^{1}(\Omega ; \boldsymbol{V} \times Q)} \leq C \Theta_{L}$.

Remark 6.1 We choose $N_{l}$ and $h_{l}$ such that their contributions to the error bounds in Theorem 6.2 are of the same order. If the norms $\left\|\boldsymbol{u}_{N}\right\|_{L^{q}\left(\Omega ; H^{k+1}(D)^{d}\right)}$ and $\left\|p_{N}\right\|_{L^{q}\left(\Omega ; H_{*}^{k+1}(D)\right)}$ are bounded independently of $N$, this is the case for $N^{-s} \sim h^{k}$. More generally, we assume that there are constants $C$ and $r \geq 0$ such that

$$
\left\|\boldsymbol{u}_{N}\right\|_{L^{q}\left(\Omega ; H^{k+1}(D)^{d}\right)} \leq C N^{r} \text { and }\left\|p_{N}\right\|_{L^{q}\left(\Omega ; H_{*}^{k+1}(D)\right)} \leq C N^{r} .
$$


Then the truncation error and spatial discretization error are equilibrated for $N_{l} \sim$ $h_{l}^{-k /(s+r)}$.

This choice of $N_{l}$ disregards the additional power of $h_{l}$ in (6.12) and (6.13). If the two estimates in (6.14) hold with $r_{u}$ and $r_{p}$, respectively, then the truncation error and spatial discretization error are equilibrated for $N_{l} \sim h_{l}^{-v}$ with $v=\min \left(\frac{k}{s+r_{u}}, \frac{k+1}{s+r_{p}}\right)$ if $t>0$ and $v=\min \left(\frac{k+1}{s+r_{u}}, \frac{k}{s+r_{p}}\right)$ if $t=0$. Thus we may set $r:=\frac{k}{v}-s$, which satisfies $0 \leq r \leq \max \left(r_{u}, r_{p}\right)$.

Remark 6.2 Let $h_{l}=2^{-l} h_{0}$, which holds if the meshes $\mathcal{T}_{l}$ are constructed as regular refinements of $\mathcal{T}_{0}$. As in Remark 6.1, we set

$$
N_{l}:=\left\lceil 2^{k l /(s+r)} N_{0}\right\rceil \sim h_{l}^{-k /(s+r)}, \quad l=0,1, \ldots, L .
$$

This results in a convergence of $2^{-s k l /(s+r)}$ of the truncation of the random field combined with the mixed finite element approximation. For a parameter $\bar{M}$, we select the number of samples as

$$
M_{l}:=\left\lceil 2^{2 s k(L-l) /(s+r)} \bar{M} L\right\rceil, \quad l=0,1, \ldots, L .
$$

Then the error bound (6.12) in Theorem 6.2 is

$$
\begin{aligned}
& \left\|\mathbb{E}\left[U_{L}\right]-E^{L}[U]\right\|_{L^{2}(\Omega ; \boldsymbol{V} \times Q)}^{2} \\
& \quad \leq C\left(\frac{1}{\bar{M} L} 2^{-2 s k L /(s+r)}+\frac{\left(N_{0}^{-s}+h_{0}^{k} N_{0}^{r}\right)^{2}}{\bar{M} L} \sum_{l=1}^{L} 2^{-2 s k(L-l) /(s+r)} 2^{-2 s k l /(s+r)}\right) \\
& \quad=C \bar{M}^{-1}\left(L^{-1}+\left(N_{0}^{-s}+h_{0}^{k} N_{0}^{r}\right)^{2}\right) 2^{-2 s k L /(s+r)} .
\end{aligned}
$$

Adding the error contribution (6.13), we see that

$$
\begin{aligned}
& \left\|\mathbb{E}[U]-E^{L}[U]\right\|_{L^{2}(\Omega ; \boldsymbol{V} \times Q)} \\
& \quad \leq C\left(\bar{M}^{-1} L^{-1}+\left(1+\bar{M}^{-1}\right)\left(N_{0}^{-s}+h_{0}^{k} N_{0}^{r}\right)^{2}\right)^{1 / 2} 2^{-s k L /(s+r)} .
\end{aligned}
$$

Thus the total error is on the order of $h_{L}^{k} N_{L}^{r}, N_{L}^{-s}$ and $M_{0}^{-1 / 2}$.

Remark 6.3 We assume that the computational cost of the numerical solution of a sample of (5.3) is $W(h) \sim h^{-\sigma d}$, i.e. the linear solver may be suboptimal. Then by (6.10), the computational cost of MLMC-MFEM with the parameters from Remark 6.2 is

$$
W^{L} \sim 2^{2 s k L /(s+r)} L \bar{M}\left(N_{0} h_{0}^{-d} \sum_{l=0}^{L} 2^{l(d-(2 s-1) k /(s+r))}+h_{0}^{-\sigma d} \sum_{l=0}^{L} 2^{l(\sigma d-2 s k /(s+r))}\right) .
$$


Thus the total work can be decomposed as $W^{L}=W_{\mathrm{KL}}^{L}+W_{\mathrm{FE}}^{L}$ with $W_{\mathrm{KL}}^{L}$, which represents the cost of the summation of the Karhunen-Loève series, equivalent to

$$
W_{\mathrm{KL}}^{L} \sim \bar{M} N_{0} h_{0}^{-d} \begin{cases}2^{2 s k L /(s+r)} L & \text { if } d<(2 s-1) k /(s+r), \\ 2^{(d+k /(s+r)) L} L^{2} & \text { if } d=(2 s-1) k /(s+r), \\ 2^{(d+k /(s+r)) L} L & \text { if } d>(2 s-1) k /(s+r),\end{cases}
$$

and $W_{\mathrm{FE}}^{L}$, which represents the cost of the finite element solution of samples of (5.3), is

$$
W_{\mathrm{FE}}^{L} \sim \bar{M} h_{0}^{-\sigma d} \begin{cases}2^{2 s k L /(s+r)} L & \text { if } \sigma d<2 s k /(s+r), \\ 2^{\sigma d L} L^{2} & \text { if } \sigma d=2 s k /(s+r), \\ 2^{\sigma d L} L & \text { if } \sigma d>2 s k /(s+r) .\end{cases}
$$

If $\sigma d \geq 2 s k /(s+r), W_{\mathrm{FE}}^{L}$ is equivalent to the computational cost of a single deterministic problem, up to a factor of $L$. For $\sigma d<2 s k /(s+r), W_{\mathrm{FE}}^{L}$ is equivalent to the cost of Monte Carlo sampling alone, with no spatial discretization. Similarly, $W_{\mathrm{KL}}^{L}$ is equal to the cost of computing a single realization of the random field, using $N_{L}$ terms of the series, or Monte Carlo for a scalar problem, whichever is more expensive, up to a constant and a factor of $L$. We note that, if $d \geq k \max (2 s-1,2 s / \sigma) /(s+r)$, then the total computational cost $W^{L}=W_{\mathrm{KL}}^{L}+W_{\mathrm{FE}}^{L}$ of MLMC-MFEM is equivalent to that of one realization of the random field with $N_{L}$ terms plus the finite element solution of a single deterministic Brinkman problem, up to a logarithmic factor. In any case, the cost $W^{L}$ of MLMC-MFEM compares favorably to that of MC-MFEM, which by Theorem 6.1 is on the order of

$$
2^{(d+k(2 s+1) /(s+r)) L}+2^{(\sigma d+2 s k /(s+r)) L} \quad \text { as } \quad L \rightarrow \infty
$$

since $h=h_{L}=2^{-L} h_{0}$ entails $N=\left\lceil 2^{k L /(s+r)} N_{0}\right\rceil$ and $M=\left\lceil 2^{2 s k L /(s+r)} M_{0}\right\rceil$ in order to equilibrate the error contributions in (6.4).

Remark 6.4 The derivation in Remark 6.3 of the total computational cost provides insight on the distribution of the work load among the discretization levels $l=0, \ldots, L$ since the terms $2^{l(d-(2 s-1) k /(s+r))}$ and $2^{l(\sigma d-2 s k /(s+r))}$ in (6.19) represent the computations performed on level $l$. Clearly, the latter term is the same for all levels if $\sigma d=2 s k /(s+r)$. For $\sigma d>2 s k /(s+r)$, the computational cost of finite element solves is dominated by the finest discretization level, and the work per level increases exponentially in $l$. If $\sigma d<2 s k /(s+r)$, more work is done on coarse discretization levels, and the work per level decreases exponentially in $l$. Similar considerations apply to the first term, which is the same on all levels if $d=(2 s-1) k /(s+r)$. We note that if $\sigma>2 s /(2 s-1)$, it is possible for the first term to decrease in $l$ but for the second to increase in $l$, i.e. for the total work required by computing realizations of the random field to be dominated by the coarsest discretization while the total cost of finite element solves is maximal on the finest mesh. 
Remark 6.5 The error bound from Theorem 6.2 induces confidence bounds for MLMC-MFEM. By Chebyshev's inequality,

$$
\mathbb{P}\left(\left\|\mathbb{E}\left[U_{L}\right]-E^{L}[U]\right\| \boldsymbol{V} \times Q \geq \eta\right) \leq \frac{1}{\eta^{2}}\left\|\mathbb{E}\left[U_{L}\right]-E^{L}[U]\right\|_{L^{2}(\Omega ; \boldsymbol{V} \times Q)}^{2} \quad \forall \quad \eta>0 .
$$

Equivalently,

$$
\mathbb{P}\left(\left\|\mathbb{E}\left[U_{L}\right]-E^{L}[U]\right\| \boldsymbol{V} \times Q \leq \frac{1}{\sqrt{\epsilon}}\left\|\mathbb{E}\left[U_{L}\right]-E^{L}[U]\right\|_{L^{2}(\Omega ; \boldsymbol{V} \times Q)}\right) \geq 1-\epsilon \quad \forall \epsilon>0 .
$$

Comparing with (6.17), we see that the $\bar{M}$ should be chosen on the order of $\epsilon^{-2}$, independently of the number of discretization levels, to ensure a failure probability of at most $\epsilon$. Then the number of samples on the finest level $M_{L}=\bar{M} L$ scales as $\epsilon^{-2} L$.

Remark 6.6 The assumption of uniform boundedness or algebraic increase of the norms $\left\|\boldsymbol{u}_{N}\right\|_{L^{q}\left(\Omega ; H^{k+1}(D)^{d}\right)}$ and $\left\|p_{N}\right\|_{L^{q}\left(\Omega ; H_{*}^{k+1}(D)\right)}$ in Remark 6.1 is realistic. For example, if $t>0, g=0$ and $\boldsymbol{f} \in L^{2}(D)^{d}$, then $\boldsymbol{u}_{N}$ and $p_{N}$ solve the Stokes problem

$$
\left\{\begin{array}{l}
-t^{2} \Delta \boldsymbol{u}_{N}+\nabla p_{N}=\boldsymbol{f}-\boldsymbol{M}_{N} \boldsymbol{u}_{N} \\
\operatorname{div} \boldsymbol{u}_{N}=0
\end{array}\right.
$$

with homogeneous boundary conditions $\boldsymbol{u}_{N}=\mathbf{0}$ on $\partial D$. We note that due to Lemma 4.2 and Lemma 4.3 , for any $q \in[1, \infty)$,

$$
\left\|\boldsymbol{M}_{N} \boldsymbol{u}_{N}\right\|_{L^{q}\left(\Omega ; L^{2}(D)^{d}\right)} \leq C\|\boldsymbol{f}\| \boldsymbol{V}^{*} \leq C\|\boldsymbol{f}\|_{L^{2}(D)^{d}}
$$

with a constant independent of $N$. If $D$ is a convex polygon in $\mathbb{R}^{2}$, then the regularity result [16, Theorem 2] implies

$$
t^{2}\left\|\boldsymbol{u}_{N}\right\|_{L^{q}\left(\Omega ; H^{2}(D)^{2}\right)}+\left\|p_{N}\right\|_{L^{q}\left(\Omega ; H_{*}^{1}(D)\right)} \leq C\|\boldsymbol{f}\|_{L^{2}(D)^{2}} .
$$

Thus $r_{u}=0$ for $k=1$ in Remark 6.1, and we may set $r=0$ provided that $r_{p} \leq s$, making use of the additional factor of $h_{l}$ in (6.12) and (6.13). Similar regularity results for polyhedral domains in three dimensions are given in [9].

Remark 6.7 For the case $t=0$, it is also realistic to expect algebraic growth in (6.14). For example, for $\boldsymbol{f}=\mathbf{0}, \boldsymbol{u}_{N}=-\boldsymbol{M}_{N}{ }^{-1} \nabla p_{N}$, and therefore $p_{N}$ is the solution of $-\operatorname{div}\left(\boldsymbol{M}_{N}^{-1} \nabla p_{N}\right)=g$ on $D$ and $\left(\boldsymbol{M}_{N}^{-1} \nabla p_{N}\right) \cdot \boldsymbol{n}=0$ on $\partial D$. We assume for simplicity that each $\boldsymbol{\Psi}_{n}$ is a scalar function multiplied by the identity matrix. Then we have

$$
-\Delta p_{N}=\boldsymbol{M}_{N} g+\boldsymbol{M}_{N} \nabla \boldsymbol{M}_{N}^{-1} \cdot \nabla p_{N}
$$


By similar arguments as in the proof of Theorem 4.1, it can be shown that

$$
\left\|\sum_{n=1}^{N} Y_{n} \nabla \boldsymbol{\Psi}_{n}\right\|_{L^{q}\left(\Omega ; L^{\infty}(D)^{d}\right)} \leq C\left(\sum_{n=1}^{N}\left|\boldsymbol{\Psi}_{n}\right|_{W^{1, \infty}(D)}^{2(1-\vartheta)}\left\|\boldsymbol{\Psi}_{n}\right\|_{W^{2, \infty}(D)}^{2 \vartheta}\right)^{1 / 2}
$$

for any $q \in[1, \infty)$ and $\vartheta \in(0,1)$. If $D$ is a convex domain, using (2.22) and Lemma 4.3, it follows that

$$
\left\|p_{N}\right\|_{L^{q}\left(\Omega ; H_{*}^{2}(D)\right)} \leq C\left[1+\left(\sum_{n=1}^{N}\left|\boldsymbol{\Psi}_{n}\right|_{W^{1, \infty}(D)}^{2(1-\vartheta)}\left\|\boldsymbol{\Psi}_{n}\right\|_{W^{2, \infty}(D)}^{2 \vartheta}\right)^{1 / 2}\right]\|g\|_{L^{2}(D)} .
$$

In many examples, such as the model problem from Section 7 below, the right hand side of (6.30) is either uniformly bounded in $N$ or grows algebraically in $N$.

\section{Numerical computations}

In the following we verify the performance of the MLMC-MFEM method with numerical examples in $\mathbb{R}^{2}$. The physical domain $D$ is chosen as $D=[-1,1] \times[-1,1]$. We load the problem with boundary conditions only, thus $g=0$ and $\boldsymbol{f}=\mathbf{0}$. By choosing Dirichlet boundary data

$$
\boldsymbol{u}_{D}=\nabla\left(r^{\beta} \sin (\beta \varphi)\right)
$$

in polar coordinates $(r, \varphi)$, we have $\boldsymbol{u}_{D} \in\left[H^{\beta}(D)\right]^{d}$ and for the boundary trace it holds $\left.u_{D}\right|_{\partial D} \in\left[H^{\beta-1 / 2}(\partial D)\right]^{d}$. The boundary conditions are enforced using Nitsche's method, cf. [15]. Thus the parameter-dependent norm $\|\boldsymbol{v}\|_{t, h}$ for the velocity is given by the expression

$$
\|\boldsymbol{v}\|_{t, h}^{2}:=\|\boldsymbol{v}\|_{t}^{2}+t^{2} \sum_{E \in \partial \mathcal{E}_{h}} \frac{1}{h_{E}}\|\llbracket v \rrbracket\|_{0, E}^{2},
$$

in which $\partial \mathcal{E}_{h}$ denotes the collection of edges residing on the boundary $\partial D$. In all of the numerical examples we choose $\beta=3.1$ to ensure sufficient regularity of the boundary data.

In the following we assume for simplicity the stochastic permeability matrix $\boldsymbol{M}$ to be isotropic, lognormal, i.e.

$$
\boldsymbol{M}(\boldsymbol{x}, \omega)=\exp (m(\boldsymbol{x}, \omega)) \boldsymbol{I}
$$

in which $m$ is an $\mathbb{R}$-valued Gaussian field on $D$ and $m(\boldsymbol{x}, \omega) \in L^{\infty}(D, \mathbb{R})$. For practical applications, such as oil reservoir simulation, the permeability is usually of this diagonal form, and thus the test case is also of practical importance. 


\subsection{Karhunen-Loève eigenpairs}

To compute the eigenpairs of the truncated Karhunen-Loève expansion (4.4) numerically, one could employ e.g. finite element methods [23]. For simplicity, we only consider analytically known eigenexpansions for the Karhunen-Loève series. We use the following result in one space dimension as a basis.

Proposition 7.1 Let $D=(-1,1), \mathbb{E}(x)=x, C\left(x, x^{\prime}\right)=\frac{\min \left\{x, x^{\prime}\right\}+1}{2} \in H^{1,1}(D \times$ $D)$. Then the eigenpairs of the Karhunen-Loève expansion are

$$
\tilde{\lambda}_{m}=\frac{8}{\pi^{2}(2 m-1)^{2}}, \quad \tilde{\varphi}_{m}(x)=\sin \left(\frac{x+1}{\sqrt{2 \lambda_{m}}}\right) .
$$

In two dimensions, we choose the eigenexpansion as the tensor product of the expansion in one dimension. Thus the truncated Karhunen-Loève expansion with $N$ terms can be written as

$$
\boldsymbol{M}_{N}(\boldsymbol{x}, \omega)=\exp \left(\sum_{m=1}^{N} Y_{m}(\omega) \sqrt{\lambda_{m}} \varphi_{m}(\boldsymbol{x})\right) \boldsymbol{I} .
$$

Here, the eigenpairs are chosen as $\lambda_{m(i, j)}=\tilde{\lambda}_{i} \tilde{\lambda}_{j}$ and $\varphi_{m(i, j)}(\boldsymbol{x})=\tilde{\varphi}_{i}\left(x_{1}\right) \tilde{\varphi}_{j}\left(x_{2}\right)$ ordered by the magnitude of the resulting eigenvalues $\left\{\lambda_{m}\right\}_{m \geq 1}$.

\subsection{Numerical experiments}

We consider three distinct test cases, each with several values of the viscosity parameter $t$. Due to the possibly low regularity of sample paths of Gaussian random fields $\boldsymbol{M}_{N}(\boldsymbol{x}, \omega)$ which, in turn, implies low regularity of the unknowns $\boldsymbol{u}$ and $p$, in all tests we employ first-order elements, i.e. $k=1$. We introduce the scaling parameter $\Theta$ for the eigenvalues, that is we modify the eigenvalues by

$$
\lambda_{m} \rightarrow \lambda_{m}^{\Theta}
$$

This gives an algebraic decay rate of $\Theta$ for the square roots of the eigenvalues, and accordingly choosing $\tau=1 / \Theta+\varepsilon$ the sequence of norms $\boldsymbol{\Psi}$ in Assumption 4.1 belongs to $\ell^{\tau}$ for every $\varepsilon>0$. Thus in Corollary 4.1 we have $s \lesssim \Theta-1 / 2$. Using the optimistic estimate $r=0$ we choose the number of terms in the Karhunen-Loève expansion on level $l$ according to (6.15) as

$$
N_{l}=N_{0} 2^{l /(\Theta-1 / 2)} .
$$

Similarly, the number of samples on level $l$ is chosen according to (6.16) as

$$
M_{l}=\bar{M} L 2^{2(L-l)} .
$$




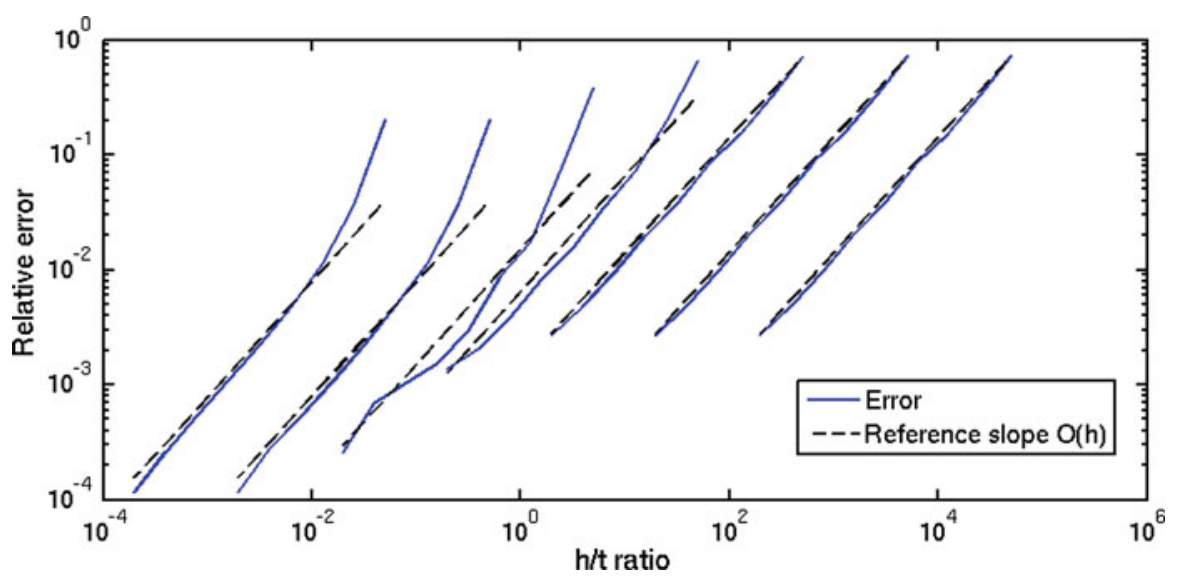

Fig. 1 Convergence in the mesh dependent norm for several values of $t$ with $\Theta=2.5$

In all of the computations we use $\bar{M}=4$ and $N_{0}=1$. As is evident from (7.6), the decay rate of the coefficients in the Karhunen-Loève expansion has a strong effect on the feasibility of the method, since evaluating a massively long series on the finer mesh levels increases the computational cost very quickly. We use an initial mesh with only five nodes for all of the computations, with the nodes located at the vertices and in the middle of the domain. The stabilization parameter $\gamma$ is chosen elementwise as proposed in Remark 5.2. Using the solution on level $L+1$ as the reference solution, we plot in Figs. 1, 4, and 7 the relative error

$$
\frac{\left\|\mathbb{E}[U]-E^{l}[U]\right\|_{L^{2}(\Omega ; \boldsymbol{V} \times Q)}}{\|\mathbb{E}[U]\|_{L^{2}(\Omega ; \boldsymbol{V} \times Q)}}
$$

for several values of the viscosity parameter $t$. In the above error expression we use for the velocity the mesh-dependent norm (7.2) and similarly for the pressure the meshdependent norm (5.8). A 12-point Gauss quadrature described in detail in [10] accurate for polynomials up to order six is used for numerical integration over triangles, and 10 Gauss integration points are used for evaluating the line integrals. We employ the Matlab backslash operator as the linear solver in all of the computations, thus we have at most $\sigma=2$ in Remark 6.3.

The computations were performed in double precision on a $866-64$ Linux platform on the HP CP4000 BL Vuori cluster at Finland's CSC ${ }^{1}$ comprising of 272 nodes with twelve $2.6 \mathrm{GHz}$ AMD Opteron cores and 16 or $32 \mathrm{~GB}$ of memory. The main program was compiled with the GCC compiler version 4.4.3 and OpenMPI 1.4.3 was used for the MPI parallelization.

\footnotetext{
1 CSC - IT Center for Science, www.csc.fi.
} 

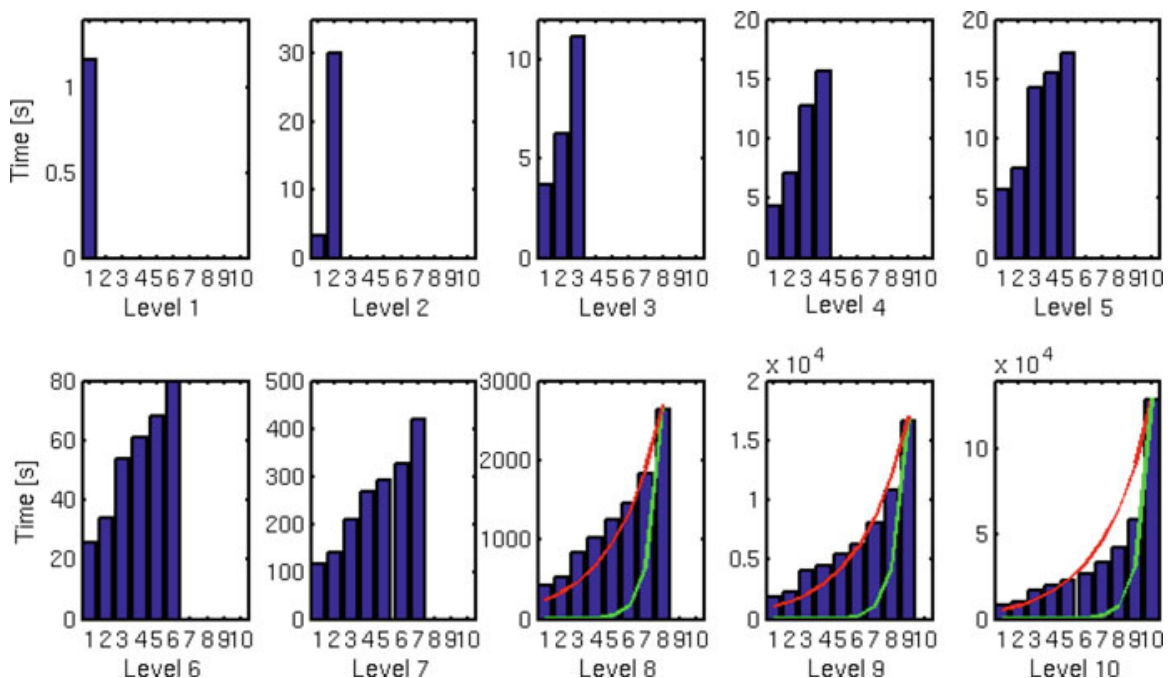

Fig. 2 Sublevel CPU time for different levels of the MLMC method with $\Theta=2.5$

\subsubsection{Series with a fast decay rate}

In the first example we choose $\Theta=2.5$. Thus the number of terms which should be retained in the Karhunen-Loève expansion to ensure a consistency error which is of the same order as the Finite Element discretization error grows as $N_{l} \sim 2^{l / 2}$. This leveldependent truncation of the Karhunen-Loève expansion allows us to test a greater number of levels. We choose $L=10$ as the reference solution with approximately 1.6 million degrees of freedom on the highest level, and test with values of $t$ ranging from $10^{-5}$ to 10 .

Referring to Fig. 1, the convergence rates predicted by Theorem 6.2 for all values of $t$ akin to the deterministic Brinkman problem [15] are attained. Furthermore, we observe that the error drops by one to two orders of magnitude as we pass numerically to the Stokes regime, where the stochastic effects of the Darcy term are suppressed by the dominating viscous Stokes term, cf. (2.1).

In Fig. 2 we plot the CPU time used on each sublevel for the MLMC-MFEM method with a total of $L$ levels. In addition, the lines in the figures represent growth relative to $2^{2 l}$ and $2^{l / 2}$, corresponding to the rates predicted in Remark 6.4 for the computation of the MFEM problem and the Karhunen-Loève series, respectively. Up to mesh level nine the computation time appears to be dominated by the computation of the Karhunen-Loève series and by the matrix assembly. However, starting from level ten there is already some evidence of the linear solver taking a more dominant part of the computational work.

In Fig. 3 we compare the CPU time of the method with the theoretical bound in Remark 6.3, as well as with the total wallclock time spent on all of the nodes used in the computation. Both observed quantities are consistent with the theoretical bound. Comparing to Remark 6.3, it is evident that the work estimate is dominated by the 


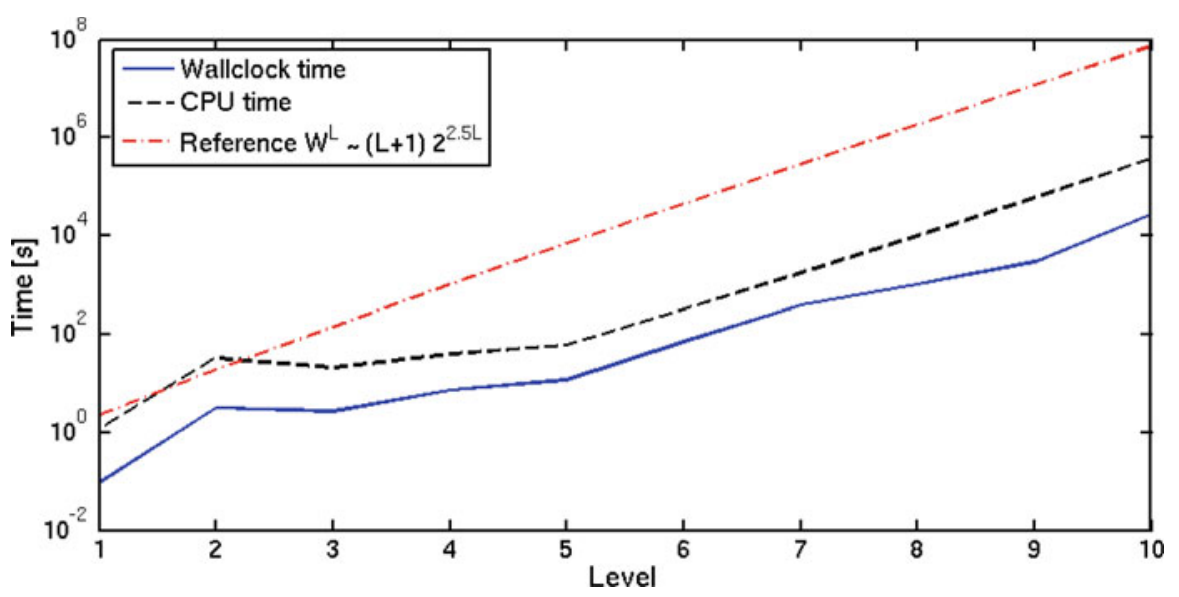

Fig. 3 Total CPU and wallclock time for different levels of the MLMC method with $\Theta=2.5$

term $W_{\mathrm{KL}}^{L}$. On the lowest levels the discrepancy is due to the relatively high amount of time spent on initialization routines, etc. Moreover, the ratio between the elapsed time and the CPU time remains approximately constant as the number of levels is increased. Thus the in-node parallelization performs equally well regardless of the number of levels.

\subsubsection{Unscaled series}

Now we consider the original series resulting from the tensor product of the one dimensional series with no scaling. This gives a rapid rate of growth for the number of terms in the Karhunen-Loève expansion, namely $N_{l} \sim 2^{2 l}$. The corresponding reference line is plotted in Fig. 5. Thus we limit the computations to using level $L=8$ as the reference solution, which results in approximately 100,000 degrees of freedom and a series with 65,536 terms on the last level.

As is evident from Fig. 4 the convergence properties of the MLMC are as predicted. One also witnesses the same improvement in the relative error upon passing into the Stokes regime. Figure 5 suggests that now the computational cost per sublevel grows as $2^{2 l}$ in accordance with Remark 6.4. This is due to the increasing cost of evaluating the Karhunen-Loève series on all of the integration points, which can be clearly seen by comparing the values to those in Fig. 2 with the same system matrix size. Also for this case the total CPU time used seems to be in good agreement with the theoretical bound of Remark 6.3, as seen in Fig. 6.

\subsubsection{Moderately scaled series}

To further test and verify the performance of the method regardless of the convergence rate of the Karhunen-Loève expansion, we test with an intermediate choice of $\Theta=1.5$ for the scaling of the eigenvalues. Here, we use the solution on level $L=9$ as the 


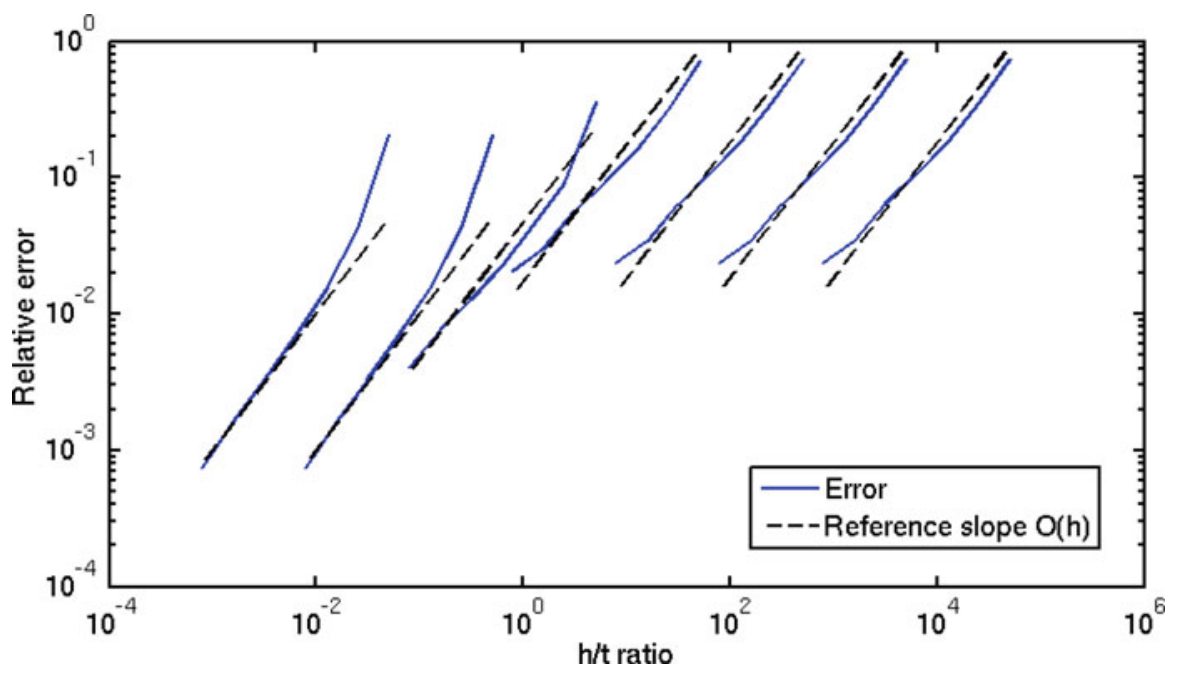

Fig. 4 Convergence in the mesh dependent norm for several values of $t$ with no scaling of the eigenvalues
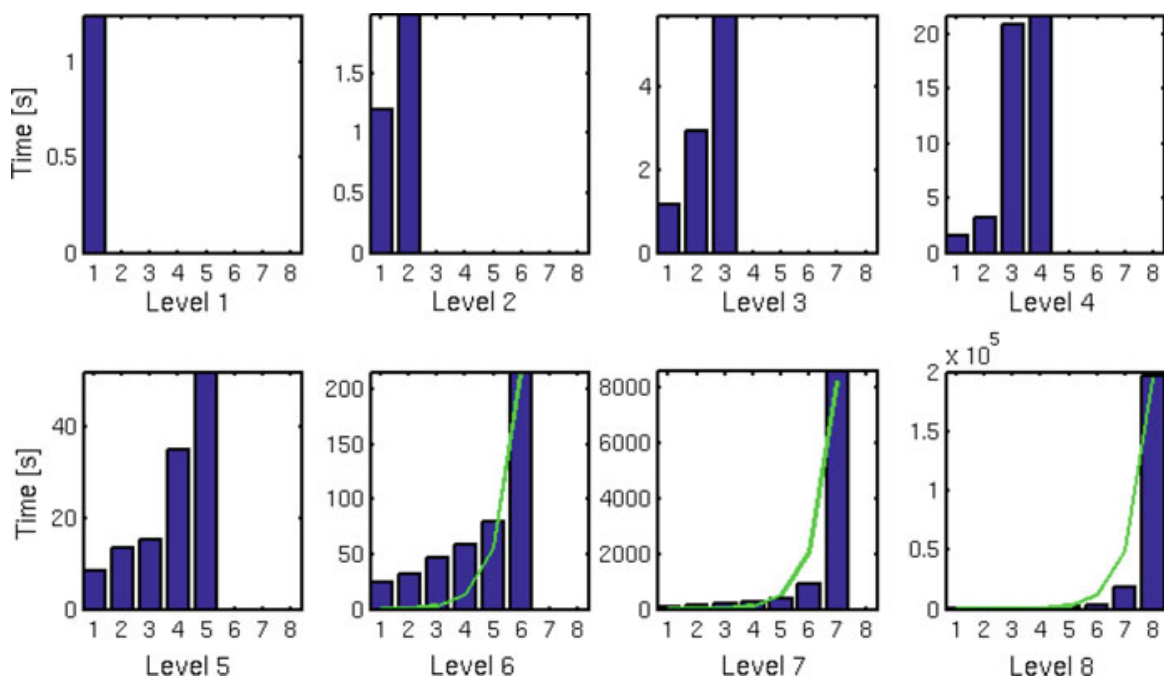

Fig. 5 Sublevel CPU time for different levels of the MLMC method with no scaling

reference solution. Figure 7 shows consistent convergence properties for the error in the mesh dependent norm compared to the previous two cases.

As for the CPU time per sublevel, the work load stemming from evaluating the Karhunen-Loève expansion is clearly reduced compared to the non-scaled KarhunenLoève expansion. Again in Fig. 8 the lines represent growth relative to $2^{2 l}$ and $2^{l}$ corresponding to the work load of the MFEM solution and computing the KarhunenLoève series, respectively. Evidently the growth rate is considerably faster than that of the Karhunen-Loève expansion with $\Theta=2.5$. As can be seen from Fig. 9, the total CPU time once again obeys the theoretical bounds given in Remark 6.3. 


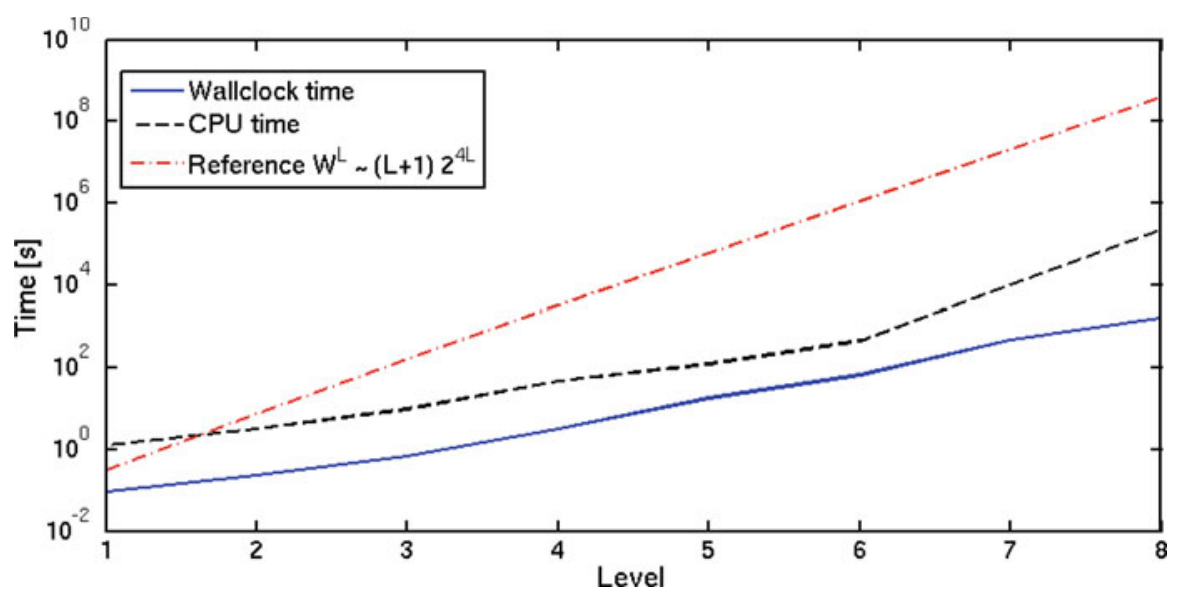

Fig. 6 Total CPU and wallclock time for different levels of the MLMC method with no scaling

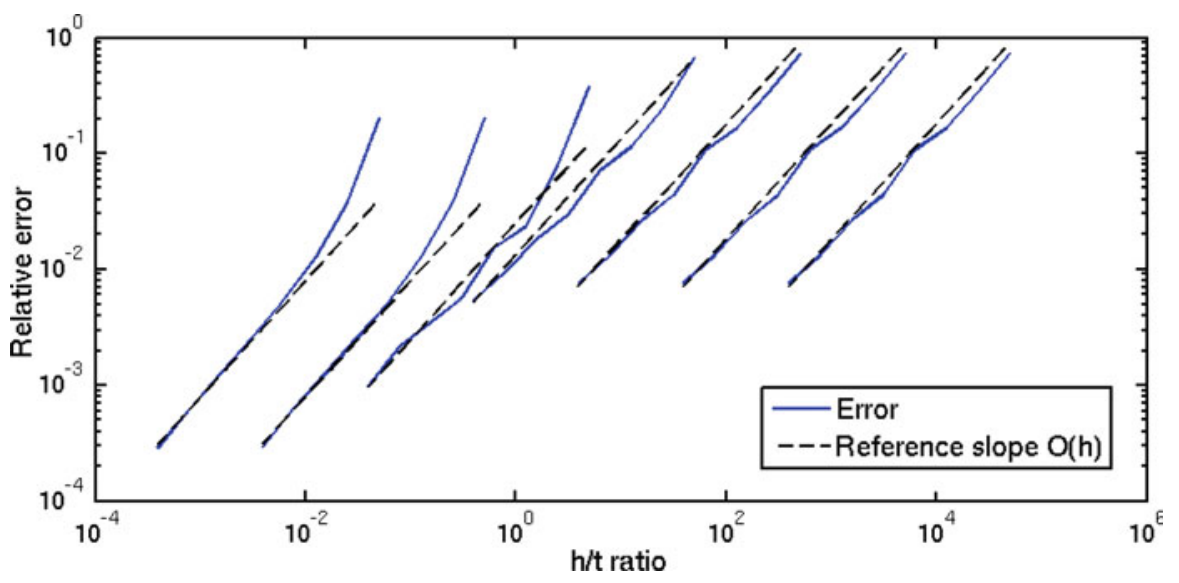

Fig. 7 Convergence in the mesh dependent norm for several values of $t$ with $\Theta=1.5$

\subsubsection{Robustness at the limit $t=0$}

We proceed to verify the performance of the MLMC Finite Element method at the limit $t=0$. We perform the test with eight levels of discretization with the above three values of eigenvalue scaling, namely $\Theta=1.0, \Theta=1.5$, and $\Theta=2.5$. As is evident from Fig. 10, the error bound of Theorem 6.2 holds also for the limiting case $t=0$ demonstrating the robustness of the method.

\subsubsection{Comparison to the single level Monte Carlo method}

Finally, we compare the calculation times for the MLMC and SLMC methods. Due to the vast number of samples required for the SLMC method on the finest mesh levels, we base the SLMC estimate on the actual computation time for a single sample on a 

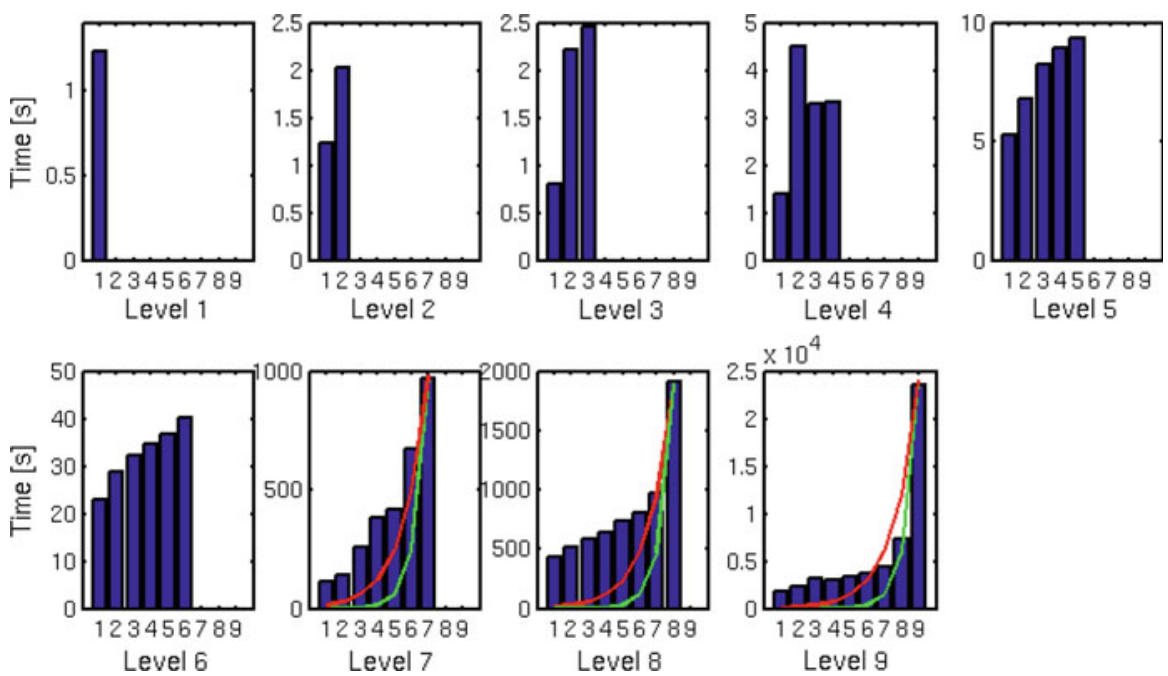

Fig. 8 Sublevel CPU time for different levels of the MLMC method $\Theta=1.5$

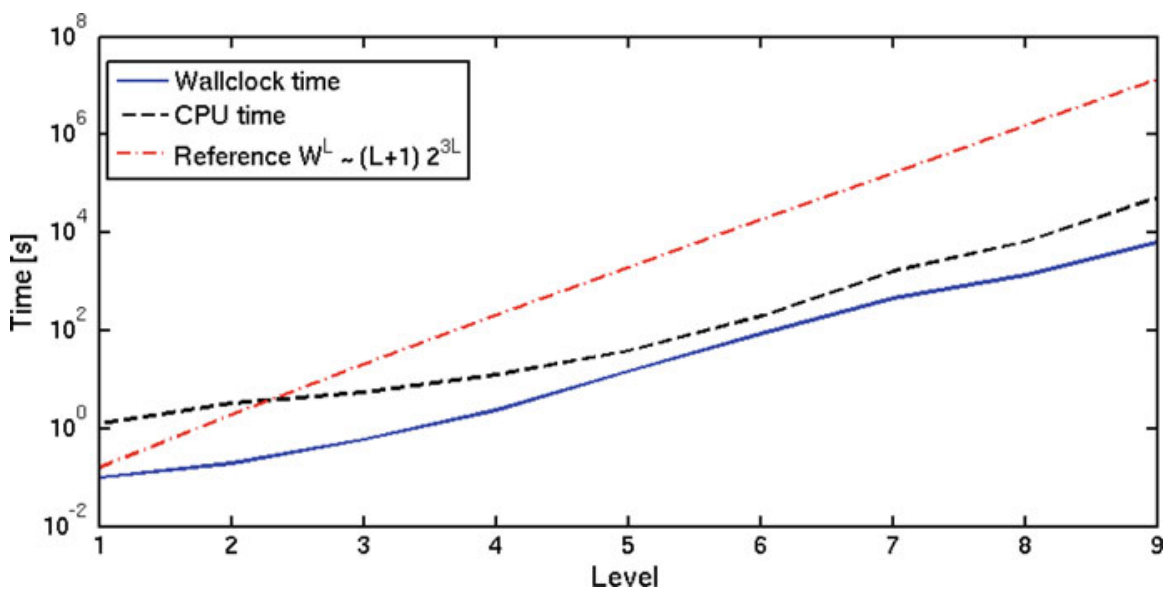

Fig. 9 Total CPU and wallclock time for different levels of the MLMC method $\Theta=1.5$

given mesh level and multiply this with the number of samples for SLMC stemming from Theorem 6.1. Thus, a comparison with Equation (6.16) gives the estimate

$$
t_{\mathrm{SLMC}} \approx \frac{t_{\mathrm{MLMC}, L}}{\bar{M} L} 2^{2 L},
$$

in which $t_{\text {MLMC,L }}$ is the total CPU time spent on level $L$. Since the tasks performed in the SLMC and MLMC are identical on a given mesh level, the estimate reflects well the actual CPU times. The results for the two highest mesh levels for each scaling parameter value $\Theta$ used are gathered in Table 1. Evidently, the number of levels used in the MLMC method has the greatest effect on the speedup attained. Nevertheless, 


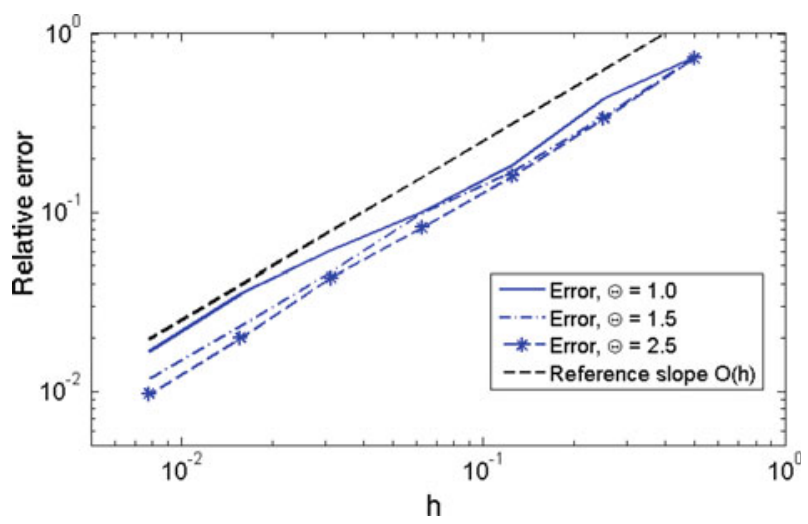

Fig. 10 Convergence in the mesh dependent norm for $t=0$

Table 1 Comparison of the MLMC and SLMC methods

\begin{tabular}{lclll}
\hline$\Theta$ and $L$ & $\begin{array}{l}\text { Total MLMC } \\
\text { CPU hours }\end{array}$ & $\begin{array}{l}\text { One sample on } L \\
\text { CPU hours }\end{array}$ & $\begin{array}{l}\text { Estimated SLMC } \\
\text { CPU hours }\end{array}$ & $\begin{array}{l}\text { Speedup } \\
\text { factor }\end{array}$ \\
\hline$\Theta=2.5, L=10$ & 101.5 & 0.892 & 935,500 & 9,220 \\
$\Theta=2.5, L=9$ & 1.68 & 0.128 & 33,490 & 2,008 \\
$\Theta=1.5, L=9$ & 14.62 & 0.181 & 47,530 & 3,250 \\
$\Theta=1.5, L=8$ & 1.839 & 0.017 & 1,083 & 589.1 \\
$\Theta=1.0, L=8$ & 62.08 & 1.704 & 111,700 & 1,799 \\
$\Theta=1.0, L=7$ & 2.940 & 0.084 & 1.388 & 472.1 \\
\hline
\end{tabular}

the speedup is also pronounced in the case of slowly converging Karhunen-Loève expansions since evaluating the Karhunen-Loève series on highly refined meshes is relatively expensive. It should be noted that for highly refined meshes SLMC simulations are out of practical reach even for this simple test case.

\subsection{Implementation and load balancing}

Monte Carlo methods are so-called embarrassingly parallel methods, i.e. during the sampling phase they achieve near optimal speedups. However, parallelizing the MLMC Finite Element method adds new technical complications. Firstly, the stiffness matrices for the problem on different mesh levels differ vastly in size, as does the number of individual problems solved per level. Accordingly, one would prefer lots of nodes with very little memory and only one core for the low-level problems, whereas for the highest levels one should have as much memory as possible. Thus, for each level the optimal division of hardware resources is different, and allocating nodes with different amounts of memory and CPU power might lead to hard-to-resolve resource starvation problems on the cluster. 
Our approach is to collect the small problems into larger batches. Specifically, we assemble several (tens to tens of thousands) problems on one node into a large matrix of approximately equal size for every level. Thus, on each level the linear system to solve is of the same order of complexity, which allows us to make use of multithreaded direct solvers inside the multicore node and take better advantage of the memory available on the individual node. Furthermore, we assemble the deterministic parts only once. There is also a considerable reduction in the internode traffic since one can sum up the results in the node and broadcast only one result vector per batch to the master MPI process, instead of communicating for each sample separately.

Another numerical complication is the numerical evaluation of a slowly converging Karhunen-Loève series. We need to evaluate the basis functions with random coefficients on a fine mesh at each integration point. Precomputing the basis functions consumes extreme amounts of memory and could not be practically implemented since the memory requirements are easily in tens or hundreds of gigabytes.

From Figs. 3, 6, and 9 one can clearly see the considerable performance gain from the internode parallelization. The Finite Element method is implemented by using a MATLAB-based Finite Element solver compiled into a standalone $\mathrm{C}$ library using the MATLAB Compiler. ${ }^{2}$ The main $\mathrm{C}$ program is a master-slave type MPI implementation. ${ }^{3}$ In addition, the numerical evaluation of the Karhunen-Loève expansion is parallelized using OpenMP in a mex-file written in C from inside the MATLAB compiled library. The normally distributed random numbers were generated using Matlab's intrinsic functions. The underlying algorithm for generating the random numbers is the well-known Mersenne Twister implemented by MathWorks in MATLAB R2010a based on the algorithm introduced in [20], and all of the subtasks divided to the nodes are seeded individually.

\section{Concluding remarks}

We presented a convergence analysis for a multi-level Monte Carlo, mixed Finite Element Method from [15] for the stochastic Brinkman problem. Owing to the logGaussian permeability tensor, statistical sampling of this tensor will, with positive probability, generate realizations which come arbitrary close to both the Stokes as well as to the Darcy limit. In the presently analyzed multi-level Monte Carlo method, the Brinkman problem is therefore discretized by a recently proposed, robust mixed Finite Element method from [15] which remains stable in the full regime, from Stokes to Darcy flow; other mixed FEMs with such robustness properties are available in [17]; in particular, the presently analyzed mixed FEM remains stable in the Darcy limit, and therefore allows also to cover these models. However, in the Darcy limit the presently proposed mixed FEM has suboptimal convergence rates in terms of the mesh width. An analogous analysis holds for the multi-level Monte Carlo $H$ (div) conforming mixed FEM analyzed recently in [17] with optimal rates (in a mesh-dependent norm) in the Darcy limit $t=0$. For the multi-level Monte Carlo FEM, the lognormal Gaussian

\footnotetext{
2 Matlab R2010a, compiler version 4.11, gec version 4.4.3.

3 OpenMPI version 1.4.3.
} 
permeability tensor was represented as an (infinite) Karhunen-Loève series with standard normal random coefficients. The present multi-level Monte Carlo convergence and complexity analysis indicated, in particular, the necessity of a level-dependent truncation of the Karhunen-Loève series, with the number of terms retained at discretization level $\ell$ chosen so as to balance the Karhunen-Loève truncation error with the discretization error at this level of discretization. The theoretical convergence analysis in the present papers states that the resulting multi-level Monte Carlo mixed FEM allows to compute approximations of the mean fields for pressure heads and flux vectors with accuracy versus work equal to that of a single, multilevel solution of the deterministic Brinkman model, uniformly over the entire range of models, from Stokes to Darcy flow. The numerical experiments support the assertions.

Acknowledgments This research was supported in part by the Swiss National Science Foundation grant No. SNF 200021-120290/1 and by the European Research Council under grant ERC AdG 247277 to CS.

\section{References}

1. Adams, R.A., Fournier, J.J.F.: Sobolev spaces, Pure and Applied Mathematics (Amsterdam), vol. 140, 2nd edn. Elsevier/Academic Press, Amsterdam (2003)

2. Allaire, G.: Homogenization of the Navier-Stokes equations in open sets perforated with tiny holes. I. Abstract framework, a volume distribution of holes. Arch. Rational Mech. Anal. 113(3), 209-259 (1990). doi:10.1007/BF00375065

3. Allaire, G.: Homogenization of the Navier-Stokes equations in open sets perforated with tiny holes. II. Noncritical sizes of the holes for a volume distribution and a surface distribution of holes. Arch. Rational Mech. Anal. 113(3), 261-298 (1990). doi:10.1007/BF00375066

4. Barth, A., Schwab, C., Zollinger, N.: Multi-level Monte Carlo finite element method for elliptic PDEs with stochastic coefficients. Numerische Mathematik 119, 132-161 (2011)

5. Bogachev, V.I.: Gaussian measures, Mathematical Surveys and Monographs, vol. 62. American Mathematical Society, RI (1998)

6. Brezzi, F., Fortin, M.: Mixed and hybrid Finite Element Methods, Springer Series in Computational Mathematics, vol. 15. Springer, New York (1991)

7. Charrier, J.: Strong and weak error estimates for elliptic partial differential equations with random coefficients. SIAM J. Numer. Anal. 50(1), 216-246 (2012)

8. Charrier, J., Scheichl, R., Teckentrup, A.: Finite element error analysis of elliptic PDEs with random coefficients and its application to multilevel Monte Carlo methods. Tech. Rep. 2, Bath Institute for, Complex Systems (2011)

9. Dauge, M.: Stationary Stokes and Navier-Stokes systems on two- or three-dimensional domains with corners. I. Linearized equations. SIAM J. Math. Anal. 20(1), 74-97 (1989). doi:10.1137/0520006

10. Dunavant, D.A.: High degree efficient symmetrical gaussian quadrature rules for the triangle. Int. J. Numer. Methods Eng. 21(6), 1129-1148 (1985). doi:10.1002/nme.1620210612

11. Fernique, X.: Intégrabilité des vecteurs gaussiens. C. R. Acad. Sci. Paris Sér. A-B 270, A1698-A1699 (1970)

12. Franca, L.P., Stenberg, R.: Error analysis of Galerkin least squares methods for the elasticity equations. SIAM J. Numer. Anal. 28(6), 1680-1697 (1991). doi:10.1137/0728084

13. Galvis, J., Sarkis, M.: Approximating infinity-dimensional stochastic Darcy's equations without uniform ellipticity. SIAM J. Numer. Anal. 47(5), 3624-3651 (2009). doi:10.1137/080717924

14. Gittelson, C.J.: Stochastic Galerkin discretization of the log-normal isotropic diffusion problem. Math. Models Methods Appl. Sci. 20(2), 237-263 (2010). doi:10.1142/S0218202510004210

15. Juntunen, M., Stenberg, R.: Analysis of finite element methods for the Brinkman problem. Calcolo 47, 129-147 (2010). doi:10.1007/s10092-009-0017-6

16. Kellogg, R.B., Osborn, J.E.: A regularity result for the Stokes problem in a convex polygon. J. Funct. Anal. 21(4), 397-431 (1976) 
17. Könnö, J., Stenberg, R.: Analysis of $H($ div)-elements for the Brinkman problem. Math. Models Methods Appl. Sci. 21, 2227-2248 (2011)

18. Könnö, J., Stenberg, R.: Numerical computations with $H$ (div)-elements for the Brinkman problem. Comput. Geosci. 2011, 1-20 (2011). doi:10.1007/s10596-011-9259-x

19. Lovadina, C., Stenberg, R.: Energy norm a posteriori error estimates for mixed finite element methods. Math. Comp. 75(256), 1659-1674 (2006). doi:10.1090/S0025-5718-06-01872-2

20. Matsumoto, M., Nishimura, T.: Mersenne twister: a 623-dimensionally equidistributed uniform pseudo-random number generator. ACM Trans. Model. Comput. Simul. 8(1), 3-30 (1998). doi:10. $1145 / 272991.272995$

21. Rajagopal, K.R.: On a hierarchy of approximate models for flows of incompressible fluids through porous solids. Math. Models Methods Appl. Sci. 17(2), 215-252 (2007). doi:10.1142/ S0218202507001899

22. Schwab, C., Gittelson, C.J.: Sparse tensor discretization of high-dimensional parametric and stochastic PDEs. In: Acta Numerica, Acta Numer., vol. 20, pp. 291-467. Cambridge Univ. Press, Cambridge (2011)

23. Schwab, C., Todor, R.A.: Karhunen-Loève approximation of random fields by generalized fast multipole methods. J. Comput. Phys. 217(1), 100-122 (2006)

24. Teckentrup, A.L., Scheichl, R., Giles, M.B., Ullmann, E.: Further analysis of multilevel monte carlo methods for elliptic pdes with random coefficients (2012). ArXiv:1204.3476v1 